\title{
New species of Asphondylia (Diptera, Cecidomyiidae, Asphondyliini) from Brazil
}

\author{
Valéria Cid Maia' \& Lázaro Araújo Oliveira²
}

${ }^{1}$ Universidade Federal do Rio de Janeiro (UFRJ), Museu Nacional (MN), Departamento de Entomologia. Rio de Janeiro, RJ, Brasil. ORCID: http://orcid.org/0000-0001-9396-5618. E-mail: maiavcid@acd.ufrj.br

2 Faculdade de Filosofia, Ciências e Letras do Alto São Francisco (FASF). Luz, MG, Brasil. http://orcid.org/0000-0002-6293-5915. E-mail: araujo.lazaro@gmail.com

\begin{abstract}
Threenew species of Asphondylia are described from Minas Gerais (Brazil):Asphondylia ingaiensis, A. rufae and A.zeyheriae. They induce galls on Moquiniastrum barrosoae (Asteraceae), Bauhinia rufa (Fabaceae), and Zeyheria montana (Bignoniaceae), respectively, all native to Brazil, being the last endemic. Illustrations of relevant morphological characters are provided. The new species are compared with congeneric Neotropical species. Types are deposited in the Museu Nacional, Entomological Collection.
\end{abstract}

Keywords. Gall midges; Host plant; Morphology; Taxonomy.

\section{INTRODUCTION}

Asphondyliini is a cosmopolitan and monophyletic tribe of Cecidomyiidae (Dorchin et al., 2019), easily recognizable by female and male characters. The female seventh sternite and eighth tergite are unique, the former is much longer than the sixth sternite and the latter is strongly sclerotized, wide, and laterally notched. Male gonostyli are short, compact, and dorsally disposed. This tribe comprises more than 500 gall-inducing species of 43 genera. It is divided in three subtribes, Asphondyliina, Polystesphina and Schizomyiina (Gagné \& Jaschhof, 2021).

Asphondylia Loew, 1850 belongs to the tribe Asphondyliina and it is morphologically characterized by a needlelike ovipositor, two-toothed gonostylus, three-segmented palpi and presence of upper and lower horns on the pupal frons (Gagné, 1994). This cosmopolitan genus is one of the most diverse of Cecidomyiidae, with about 300 species. All are gall-inducers and together they are associated with more than 65 plant families (Gagné \& Jaschhof, 2021). In Brazil, 22 described species are known.

In this paper, three new species of Asphondylia are described: $A$. ingaiensis, $A$. rufae, and A. zeyheriae. The first induces galls on Moquiniastrum barrosoae (Cabrera) G. Sancho (Asteraceae), the second on Bauhinia rufa (Bong.) Steud. (Fabaceae), and the third on Zeyheria montana Mart. (Bignoniaceae).

The neotropical genus Moquiniastrum (Cabrera) G. Sancho occurs only in South America.
It is known from 21 species, 19 of them reported in Brazil (Funk et al., 2014; Sancho, 2020). Up to date, a single cecidomyiid species has been associated with this plant genus, Asphondylia gochnatiae Maia, 2008 (Diptera, Cecidomyiidae), inducing galls on Moquiniastrum barrosoae (Cabrera) G. Sancho, a native plant to Brazil, where it occurs only in Cerrado areas (Sancho, 2020).

The pantropical genus Bauhinia L. is known from 300 to 350 species (Sinou et al., 2016). In Brazil, it is represented by 69 species (Vaz, 2020), including Bauhinia rufa, recorded only in Cerrado areas of two states, Goiás and Minas Gerais. Four species of gall midges induce galls on Bauhinia: Asphondylia microcapillata Maia, 2005, Schizomyia macrocapillata Maia, 2005 (both on B. brevipes Vogel), S. barreirensis Santos et al., 2019 on B. cupulata Benth., and S. tuiuiu Urso-Guimarães \& Amorim, 2002 on B. holophylla (Bong.) Steud. (Gagné \& Jaschhof, 2021; Urso-Guimarães, 2019), all described from Brazil and until this moment restricted to the country.

The Neotropical genus Zeyheria Mart. comprehends only two species, Z. tuberculosa and Zeyheria montana, the former occurring in Brazil and Bolivia, while the latter is endemic to Brazil, where it is widespread, with records from the Amazonas (North region) to Paraná (South region) (Fischer et al., 2004). So far, no gall-inducing species is known on Zeyheria.

In Brazil, Asphondylia occurs on 12 plant families. Six species induce galls on Asteraceae, 
A. cipo Urso-Guimarães, 2018, A. glomeratae Gagné, 2001, A. gochnatiae Maia, 2008, A. moehni Skuhravá 1989, A. serrata Maia, 2004, and A. ulei Rübsaamen, 1908 (Urso-Guimarães, 2018 published a key to the species on Asteraceae in the Neotropical region), and two on Fabaceae, A. microcapillata and A. sennae Maia \& Couri, 1992 (Gagné \& Jaschhof, 2021). This is the first record of Asphondylia on Bignoniaceae in Brazil.

\section{MATERIAL AND METHODS}

Galls on Moquiniastrum barrosoae (Asteraceae), Bauhinia rufa (Fabaceae), and Zeyheria montana (Bignoniaceae) were collected in different localities of the state of Minas Gerais (Southeastern Brazil): the first in Ingaí $\left(21^{\circ} 20^{\prime} 47^{\prime \prime} \mathrm{S}, 44^{\circ} 59^{\prime} 27^{\prime \prime} \mathrm{W}\right)$ by F. Faria, the second in Dores do Indaiá $\left(19^{\circ} 27^{\prime} 51^{\prime \prime} \mathrm{S}, 45^{\circ} 36^{\prime} 3^{\prime \prime} \mathrm{W}\right)$ by L.A. Oliveira and the third in Uberlândia (18 $\left.54^{\prime} 41^{\prime \prime} \mathrm{S}, 48^{\circ} 15^{\prime} 44^{\prime \prime} \mathrm{W}\right)$ and Serra do Cipó $\left(19^{\circ} 13^{\prime}, 43^{\circ} 27^{\prime}-43^{\circ} 37^{\prime} \mathrm{W}\right)$ by J.F. Andrade. Field works were performed in different dates (see "Material Examined"). Specimens were reared in lab and sent in micro vials with $70 \%$ ethanol to VCM for identification. Then, all material was prepared and mounted on microscope slides following the methods outlined in Gagné (1994). The genus was identified using the key of Gagné (1994), and the new species were proposed after comparison with literature data (host plants, gall morphology and cecidomyiid descriptions). All specimens were deposited at the Entomological Collection of the Museu Nacional/Universidade Federal do Rio de Janeiro (MNRJ).

Morphological studies and drawings were made with the aid of an optical microscope coupled to a photographic camera and drawing tube. Measurements were done using a microscope slide with scale from 0.01 to $5.0 \mathrm{~mm}$. Length of wings was measured from the arculus to the apex; total length of females from vertex to posterior margin of the $8^{\text {th }}$ abdominal segment; and length of antennae, upper and lower facial horns as indicated in Figs. 1A-C. All drawings were edited using Corel DRAW ${ }^{\circledR}$. Adult morphological terminology follows Cumming \& Wood (2009) and larval and pupal stages follows Gagné (1989).

Type material of Asphondylia gochnatiae and A. microcapillata from the Cecidomyiidae Collection of the Museu Nacional (MNRJ)/Universidade Federal do Rio de Janeiro were examined and photographed to be compared to the new species. Morphological data on A. godmanniae Möhn, 1959 were obtained from literature.

\section{RESULTS}

\section{Taxonomy}

\section{Asphondylia ingaiensis Maia, sp. nov.}

(Figs. 2-8)

Diagnosis: Male hypoproct truncated apically, deeply bilobed; ovipositor with needle part about 2.0-2.5 X length $7^{\text {th }}$ sternite; pupa: antennal horn with apical part
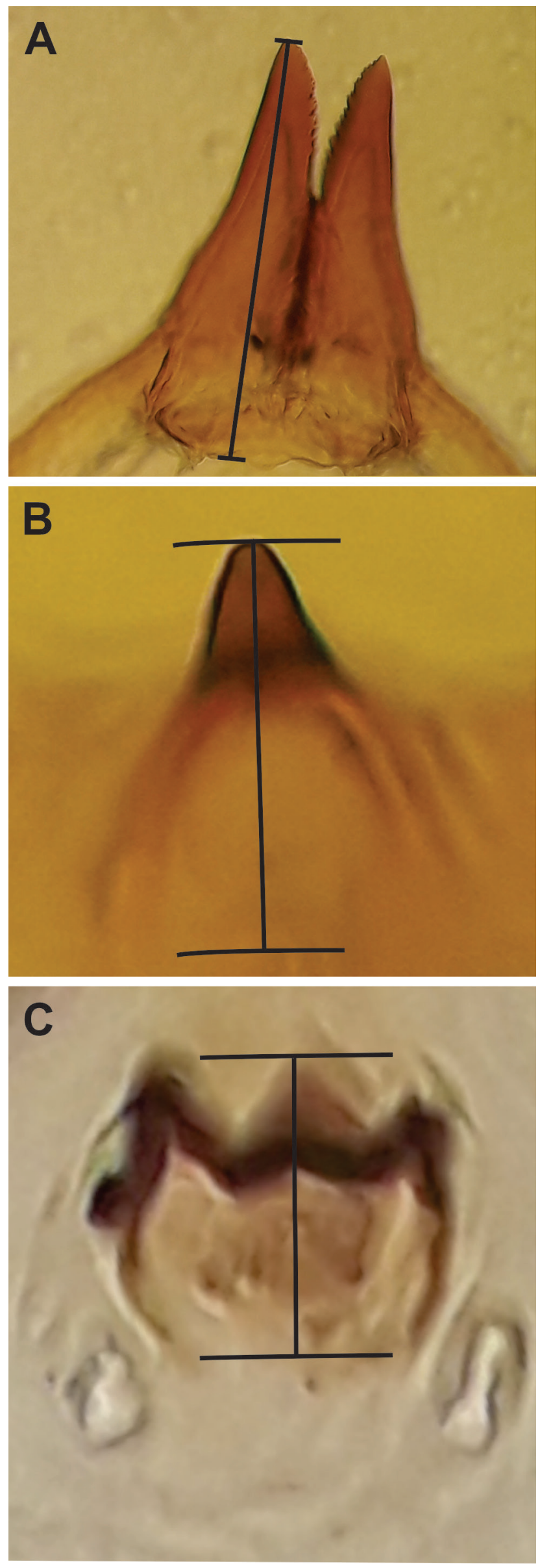

Figure 1. Length of cephalic horns indicated by black bars. (A) Antennal horns, (B) Upper facial horn, (C) Lower facial horn. 

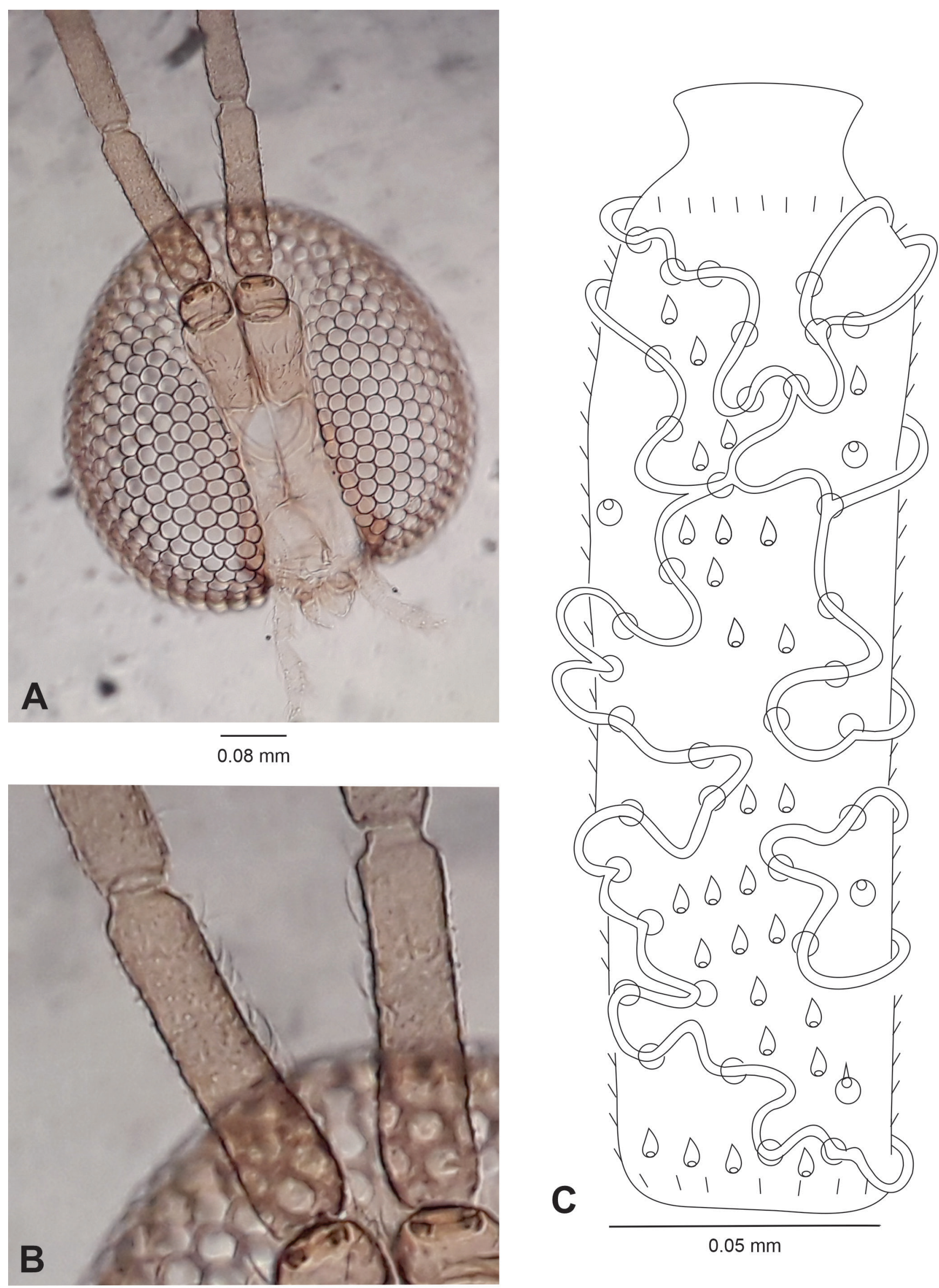

$1.20 \mathrm{~mm}$

Figure 2. Asphondylia ingaiensis sp. nov., male. (A) Head, ventral view, (B) $1^{\text {st }}$ and $2^{\text {nd }}$ flagellomeres, (C) $3^{\text {rd }}$ flagellomere. 

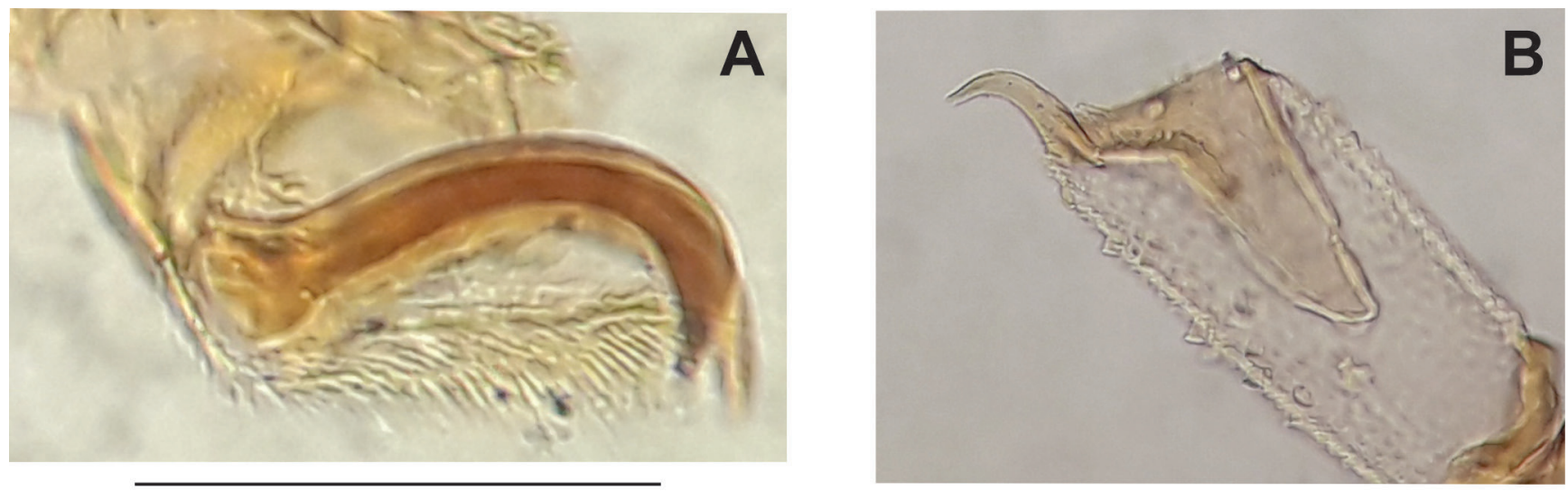

$0.05 \mathrm{~mm}$

$0.13 \mathrm{~mm}$

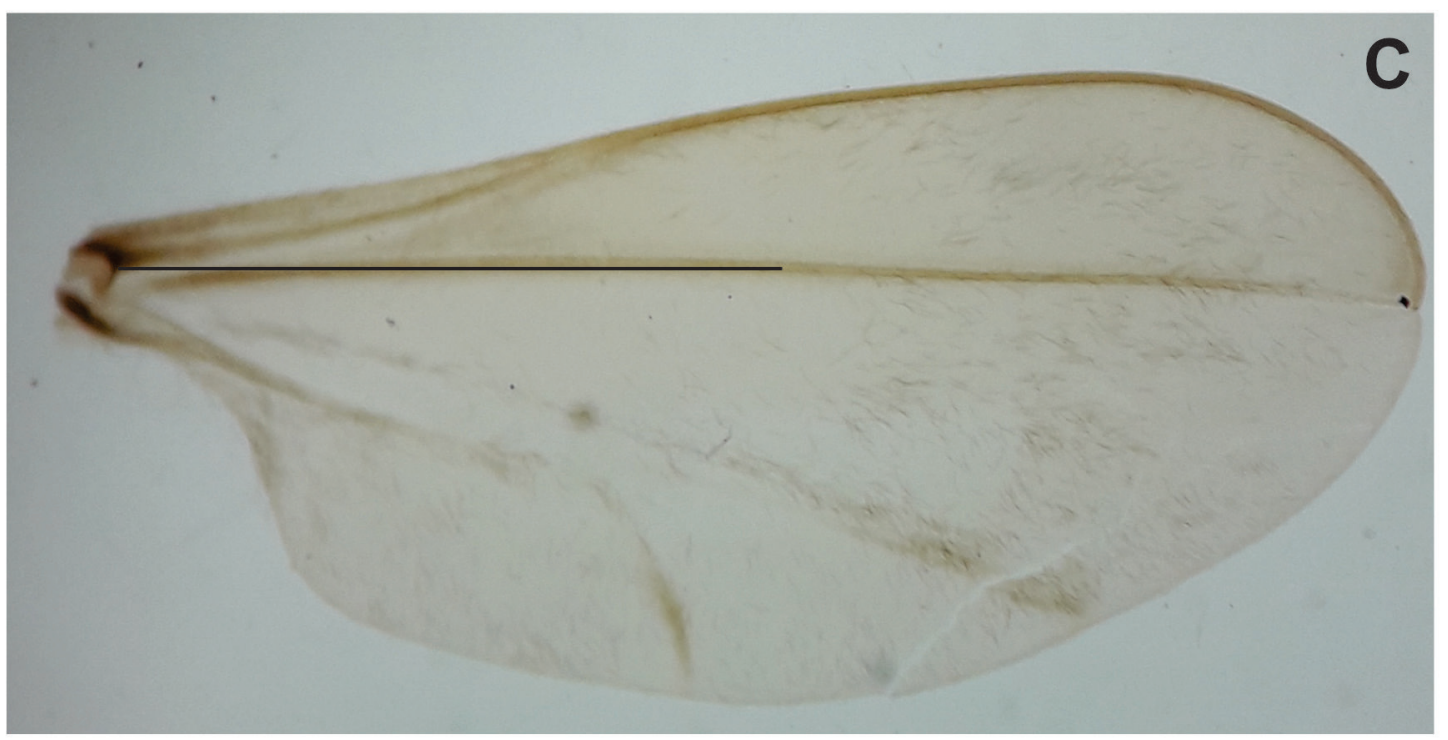

$1.50 \mathrm{~mm}$

Figure 3. Asphondylia ingaiensis sp. nov., male. (A) Foreleg, tarsal claw and empodium, lateral view (B) Midleg, first tarsomere, lateral view (C) Wing.
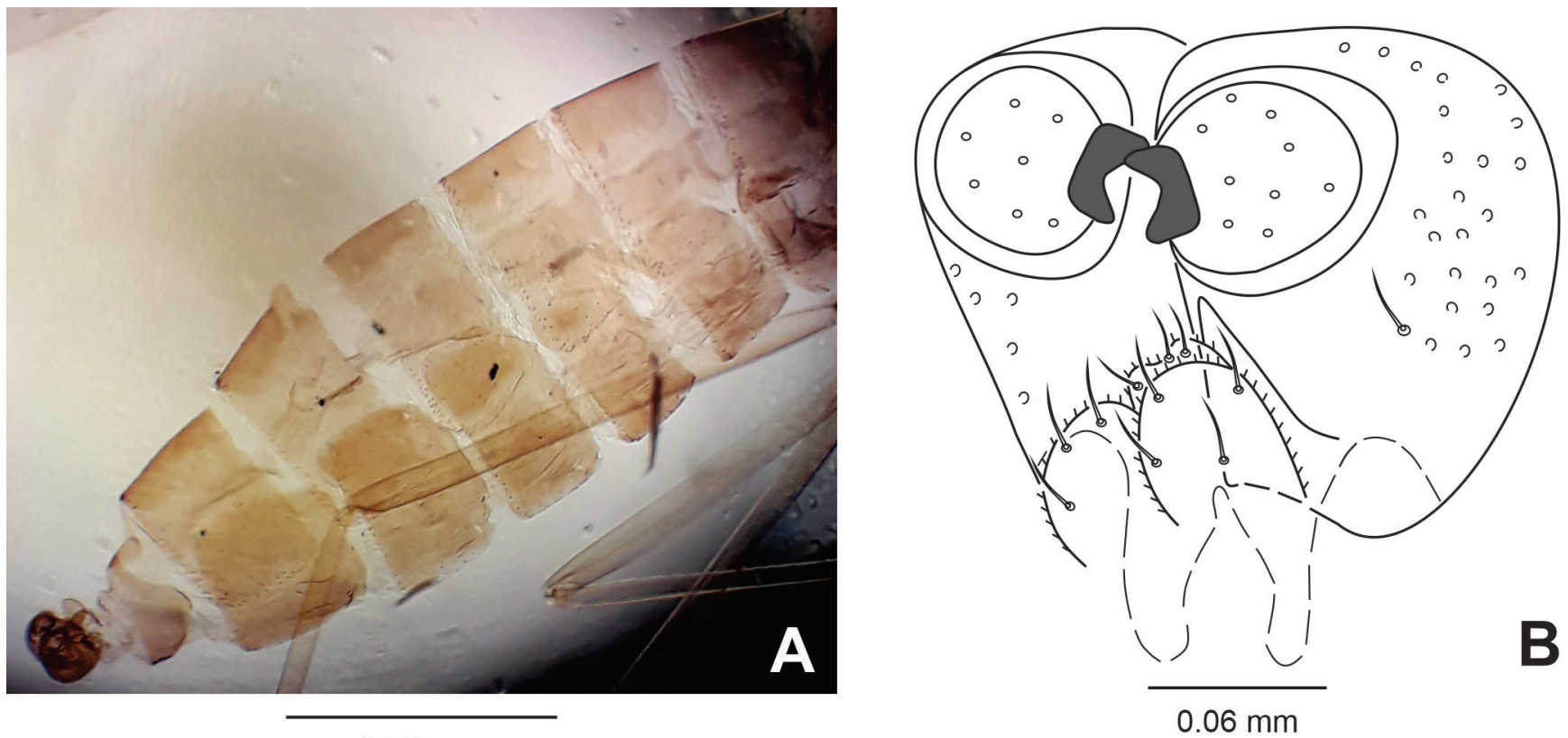

$0.70 \mathrm{~mm}$

Figure 4. Asphondylia ingaiensis sp. nov., male. (A) Abdomen, lateral view, (B) $6^{\text {th }}-8^{\text {th }}$ abdominal segments, lateral view. 
about $0.6 \mathrm{X}$ length basal part, upper frontal horn simple, $0.5 \mathrm{X}$ length antennal horn, lower frontal horn tridentate $0.3 \mathrm{X}$ length antennal horn, $8^{\text {th }}$ abdominal segment with 9-12 dorsal spines in the posterior row.

Male: Body length: 3.40-3.90 mm ( $=2)$. Head (Fig. $2 \mathrm{~A})$ : 0.50-0.55 mm long, $0.50-0.55 \mathrm{~mm}$ wide $(\mathrm{N}=2)$, eye facets circular, closely appressed; antennae: flagellomeres 1 and 2 not fused (Fig. 2B), scape truncated conical, setose, $0.15-0.14 \mathrm{~mm}$ long, $0.05 \mathrm{~mm}$ wide $(\mathrm{N}=2)$, pedicel globose, setose, $0.06 \mathrm{~mm}$ long, $0.05 \mathrm{~mm}$ wide, $1^{\text {st }} 9^{\text {th }}$ flagellomeres cylindrical, all $0.05 \mathrm{~mm}$ wide, circumfila longitudinally wavy, dense, anastomosing, equally spread along segments (Fig. 2C), $1^{\text {st }}$ flagellomere $0.22-0.24 \mathrm{~mm}$ long, $2^{\text {nd }}$ flagellomere $0.22-0.21 \mathrm{~mm}$ long, $3^{\text {rd }}$ flagellomere $0.22-0.20 \mathrm{~mm}$ long, $4^{\text {th }}-8^{\text {th }}$ flagellomeres $0.20 \mathrm{~mm}$ long, $9^{\text {th }}$ flagellomere $0.18-0.19 \mathrm{~mm}$ long, $10^{\text {th }}-12^{\text {th }}$ flagellomeres missing $(N=2)$, proportion flagellomere neck-node 1:9; frons with 30-32 setae (male, $\mathrm{N}=2$ ); mouth parts: labrum long-attenuated; hypopharynx of the same shape of labrum, with long lateral setulae, anteriorly directed; labella elongate and convex, $0.13 \mathrm{~mm}$ long, $0.04 \mathrm{~mm}$ wide $(\mathrm{N}=1)$, with lateral and mesal setae; palpus $0.33-0.39 \mathrm{~mm}$ long: $1^{\text {st }}$ segment globoid, $0.06-0.07 \mathrm{~mm}$ long, 0.04-0.05 m wide, $2^{\text {nd }}$ segment cylindrical, $0.08-0.11 \mathrm{~mm}$ long, 0.04-0.05 $\mathrm{mm}$ wide, $3^{\text {rd }}$ segment fusiform, 0.19-0.21 mm long, $0.05 \mathrm{~mm}$ wide $(\mathrm{N}=2)$, all segments with setae. Thorax: scutum with two dorsocentral rows of setae, setae more abundant anteriorly, two groups of lateral setae more abundant proximally, extending from base to distal margin, scales intermixed; scutellum with scattered setae; anepimeron setose; remaining pleural sclerites bare; legs: tarsal claws curved beyond midlength, anisomorphic, less robust on foreleg than on mid and hindlegs, empodium as long as claws (Fig. 3A); first tarsomere of each leg with an apical hook-like projection $0.03 \mathrm{~mm}$ long (Fig. 3B); wing: length 2.70-2.90 mm ( $\mathrm{N}=3$ ) (Fig. 3C). Abdomen (Fig. 4A): trichoid sensilla not visible; $1^{\text {st }} 7^{\text {th }}$

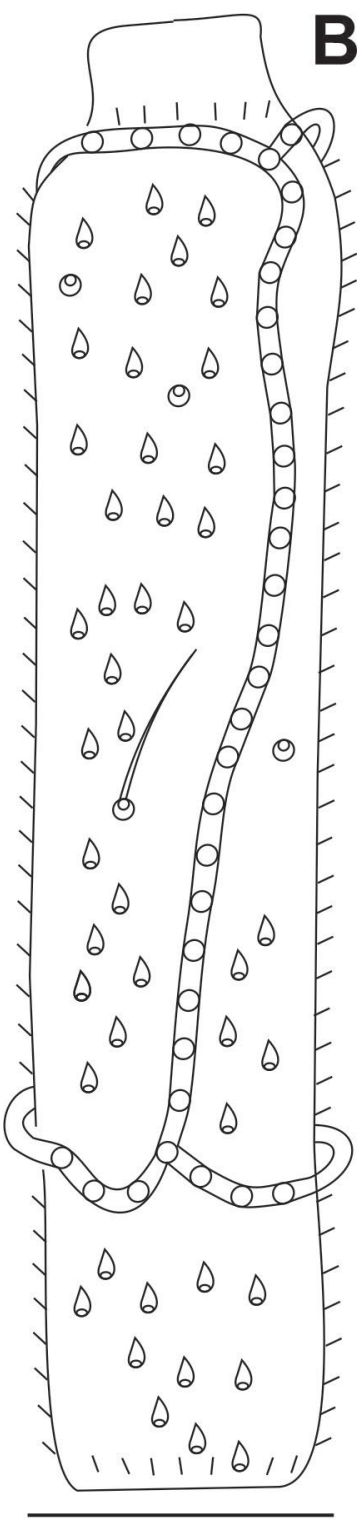

$0.05 \mathrm{~mm}$
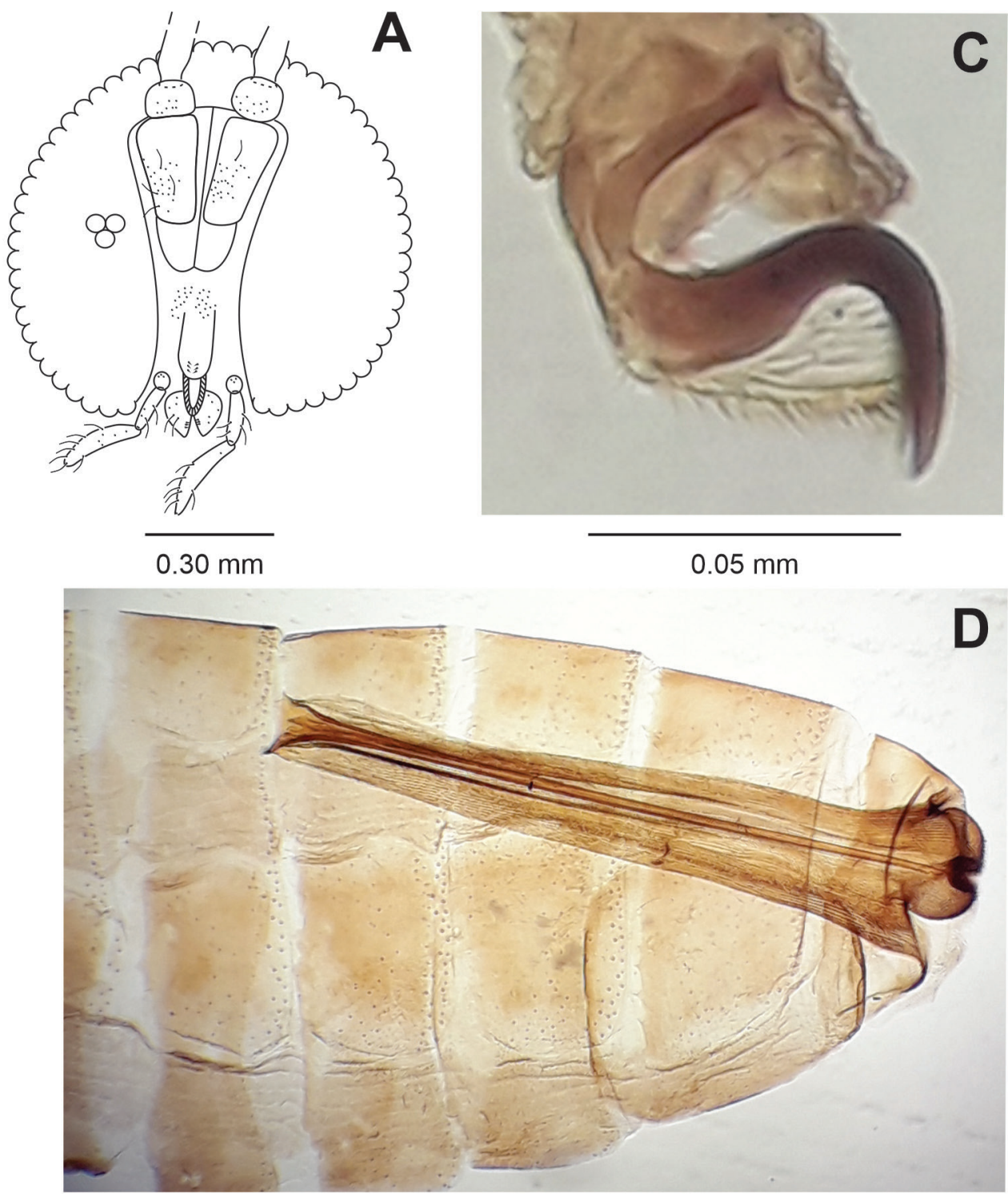

$0.80 \mathrm{~mm}$

Figure 5. Asphondylia ingaiensis sp. nov., female. (A) Head, ventral view, (B) $3^{\text {rd }}$ flagellomere, (C) midleg, tarsal claw and empodium, lateral view, (D) Abdomen ( $4^{\text {th }}$ segment to end), lateral view. 
tergites sclerotized, rectangular with a posterior row of setae, few scattered lateral setae and mostly covered elsewhere with scales, $8^{\text {th }}$ tergite bare, narrow, with mesal reentrance in the anterior margin (Fig. 4B); $2^{\text {nd }}-8^{\text {th }}$ sternites sclerotized, rectangular, narrower than tergites, with a posterior row of setae, several setae and midlength, few lateral setae, and mostly covered elsewhere with scales; $8^{\text {th }}$ sternite with setae at $2 / 3$ distal and mostly covered elsewhere with scales. Terminalia (Fig. 4B): gonocoxite short and stout, $0.16 \mathrm{~mm}$ long, $0.12 \mathrm{~mm}$ wide; gonostylus ovoid, $0.07 \mathrm{~mm}$ long, $0.04 \mathrm{~mm}$ wide, teeth $0.015 \mathrm{~mm}$ long, $0.020 \mathrm{~mm}$ wide $(\mathrm{N}=3)$; hypoproct truncated apically and deeply bilobed.

Female: Body length: $3.80-4.60 \mathrm{~mm}(\mathrm{~N}=4)$. Head (Fig. 5A): about the same as male, antennae: scape $0.15 \mathrm{~mm}$ long $(\mathrm{N}=1)$, pedicel $0.06 \mathrm{~mm}$ long $(\mathrm{N}=1)$, $1^{\text {st }} 8^{\text {th }}$ flagellomeres cylindrical, all $0.05 \mathrm{~mm}$ wide, circumfila comprising two longitudinal bands connected sub basally and apically by two transverse bands (Fig. 5B), $1^{\text {st }}$ flagellomere $0.28 \mathrm{~mm}$ long $(\mathrm{N}=1), 2^{\text {nd }}$ flagellomere $0.26 \mathrm{~mm}$ long $(\mathrm{N}=1), 3^{\text {rd }}$ flagellomere $0.25 \mathrm{~mm}$ long $(\mathrm{N}=1), 4^{\text {th }}$ flagellomere $0.23 \mathrm{~mm}$ long $(\mathrm{N}=1), 5^{\text {th }}$ flagellomere $0.24 \mathrm{~mm}$ long $(\mathrm{N}=1), 6^{\text {th }}$ flagellomere $0.22 \mathrm{~mm}$ long $(\mathrm{N}=1), 7^{\text {th }}-8^{\text {th }}$ flagellomeres $0.21 \mathrm{~mm}$ long $(\mathrm{N}=1)$, $9^{\text {th }}-12^{\text {th }}$ flagellomeres missing; mouthparts: labellum $0.07 \mathrm{~mm}$ long, $0.04 \mathrm{~mm}$ wide at midlength $(\mathrm{N}=2)$, palpus $0.22-0.24 \mathrm{~mm}$ long $(\mathrm{N}=2)$ : $1^{\text {st }}$ segment globose $0.03 \mathrm{~mm}$ long and $0.03 \mathrm{~mm}$ wide $(\mathrm{N}=2), 2^{\text {nd }}$ segment cylindrical $0.07 \mathrm{~mm}$ long, $0.02 \mathrm{~mm}$ wide at midlength $(\mathrm{N}=2), 3^{\text {rd }}$ segment claviform 0.12-0.14 $\mathrm{mm}$ long and $0.03 \mathrm{~mm}$ wide at midlength $(\mathrm{N}=2)$. Thorax: wing length: $3.00-4.80 \mathrm{~mm}$ $(\mathrm{N}=7)$; tarsal claws more sclerotized and robust than in male (Fig. 5C). Abdomen (Fig. 5D): trichoid sensil-

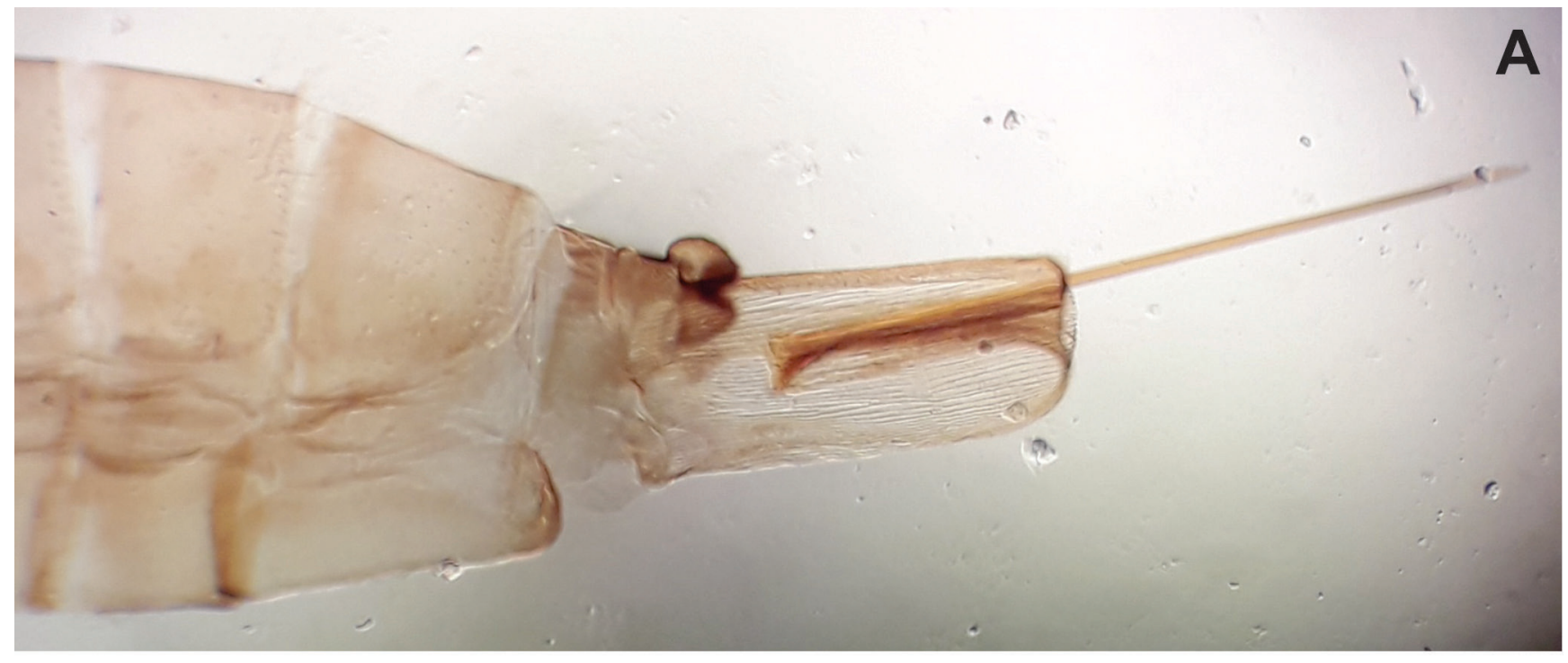

$0.40 \mathrm{~mm}$

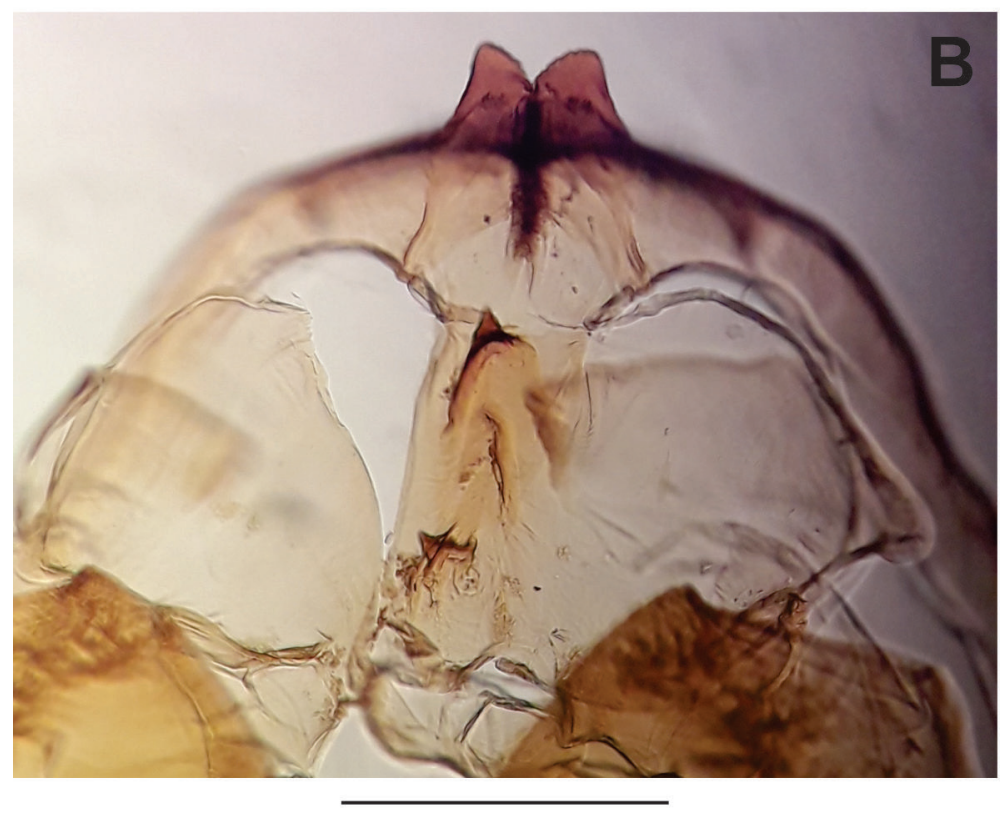

$0.34 \mathrm{~mm}$

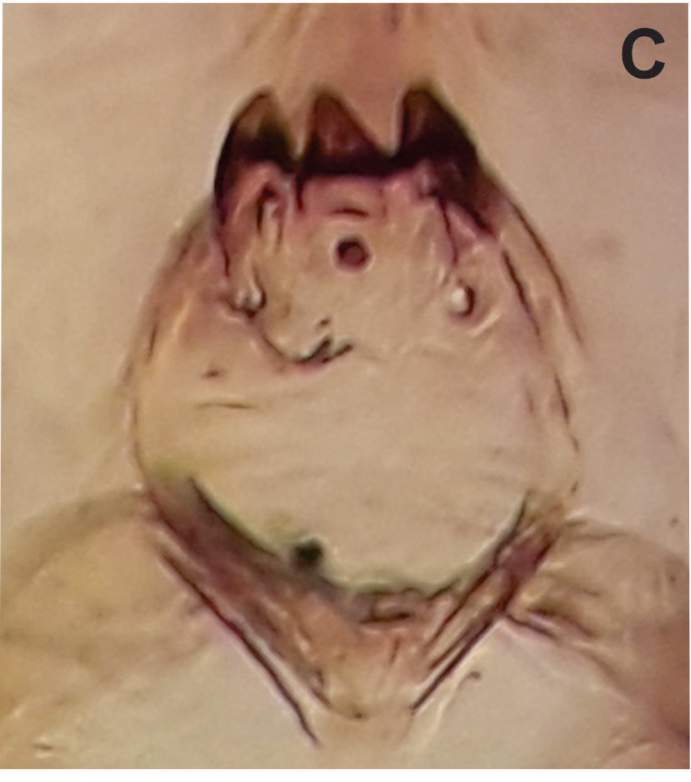

$0.06 \mathrm{~mm}$

Figure 6. Asphondylia ingaiensis sp. nov. (A) Female $6^{\text {th }}$ abdominal segment to end, lateral view, (B-C) Pupa, (B) Head, frontal view, (C) Lower facial horn. 
lae not visible, $1^{\text {st }} 7^{\text {th }}$ tergites as in male, $8^{\text {th }}$ tergite with posterior margin with lobes $0.28-0.30 \mathrm{~mm}$ long $(\mathrm{N}=3)$, $2^{\text {nd }}-6^{\text {th }}$ sternites as in male, $7^{\text {th }}$ sternite with anterior margin and posterior margins more sclerotized mesally, 0.65-0.70 mm long, 1.54-1.75 X length sternite $6(\mathrm{~N}=4)$, setose (except basally), mostly covered elsewhere with scales; sternite 8 not sclerotized; ovipositor (Fig. 6A): needle part 1.35-1.70 mm long, 2.08-2.42 X length sternite 7 $(n=4)$. Other characters as in male.

Pupa: Color: brownish. Body length: $4.20 \mathrm{~mm}(\mathrm{~N}=1)$. Head (Fig. 6B): antennal horn $0.28 \mathrm{~mm}$ long $(\mathrm{N}=1)$, triangular, with inner margin serrated; apical seta $0.04 \mathrm{~mm}$ long ( $\mathrm{N}=1)$; upper facial horn simple, $0.14 \mathrm{~mm}$ long $(\mathrm{N}=2)$, triangular; lower facial horn tridentate, $0.09 \mathrm{~mm}$ long $(\mathrm{N}=2)$, triangular (Fig. $6 \mathrm{C})$; two pairs of lower facial papillae: one pair setose, the other bare; three pairs of lateral facial papillae: one pair setose, two bare; upper cephalic margin thickened laterally. Thorax: prothoracic spiracle 0.13-0.14 mm long, as long as antennal basal width, setiform, slightly curved $(\mathrm{N}=2)$, integument wrinkly (Figs. 7A, B). Abdomen: segments 2-8 with transverse rows of crescent dorsal spines at basal half (Fig. $8 \mathrm{~A}$ ); posterior row with $23-25$ spines in the $2^{\text {nd }}$ segment, $22-25$ in the $3^{\text {rd }}, 22-24$ in the $4^{\text {th }}, 21-24$ in the $5^{\text {th }}, 18-24$ in the $6^{\text {th }}$, $13-18$ in the $7^{\text {th }}$ and $9-12$ in the $8^{\text {th }}$.

Larva: Body length: $3.20 \mathrm{~mm}(\mathrm{~N}=1)$; cephalic head $0.05 \mathrm{~mm}$ long, $0.11 \mathrm{~mm}$ wide $(\mathrm{N}=1)$. Spatula quadridentate, $0.34 \mathrm{~mm}$ long $(\mathrm{n}=1)$, lateral teeth $0.05 \mathrm{~mm}$ long,

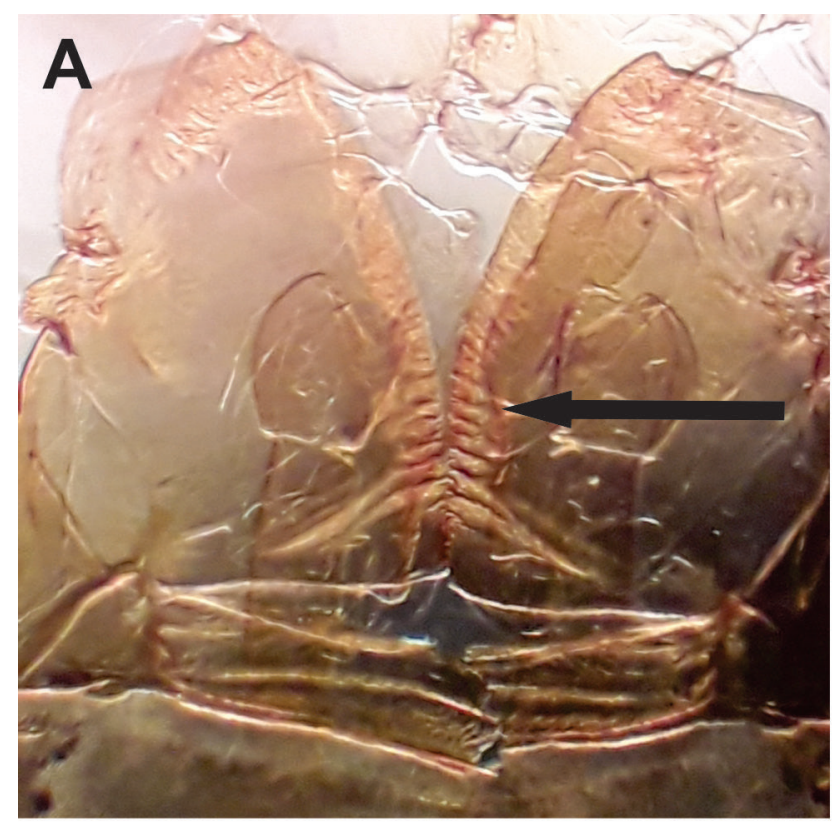

$0.60 \mathrm{~mm}$ mesal teeth $0.04 \mathrm{~mm}$ long $(\mathrm{N}=1)$ (Fig. $8 \mathrm{~B})$; four setose lateral papillae $0.01 \mathrm{~mm}$ long of each side of spatula.

Gall: On leaves, globoid, yellow, with trichomes.

Material examined: Holotype male, BRAZIL, Minas Gerais, Ingaí, Reserva Biológica Unilavras-Boqueirão, 29.XI.2013, F. Faria col. (MNRJ).

Paratypes: Same data as holotype, except: III.2007, 1 larva; 08.X.2013, 1 female and 1 pupal exuvia; 25.X.2013, 1 female and 2 pupal exuviae; 29.X.2013, 1 male, 1 female; 05.XI.2013, 1 female; 21.XI.2013, 1 pupal exuvia; 22.XI.2013, 1 female; 29.XI.2013, 1 male; 30.XII.2013, 1 male (MNRJ).

Etymology: The name "ingaiensis" refers to the type-locality, Ingaí.

Remarks: Asphondylia ingaiensis induces galls on Moquiniastrum barrosoae (Cabrera) G. Sancho (Asteraceae). Moquiniastrum hosts a second species of Cecidomyiidae, Asphondylia gochnatiae, which induces morphologically similar galls on $M$. polymorphum (Less.) G. Sancho (as Gochnatia polymorpha (Less.) Cabrera in Maia et al., 2008, a homotypical synonym). Comparing both species, we realized that the new species are have shorter flagellomeres, longer palpi, longer empodium, longer wing (specially in females), male hypoproct wider apically, shorter proportion between female $7^{\text {th }}$ sternite

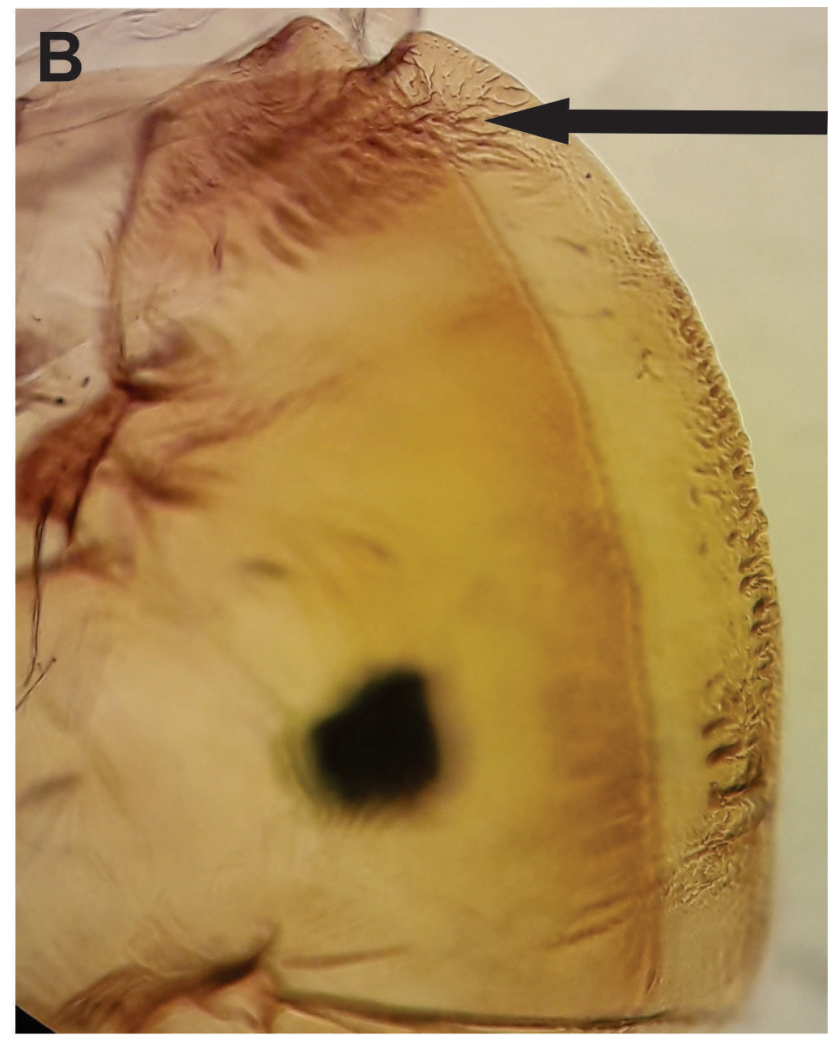

Figure 7. Asphondylia ingaiensis sp. nov., pupa, thorax integument. (A) Dorsal view, (B) Lateral view. 
length and needle part of the ovipositor. Furthermore, pupa of Asphondylia ingaiensis has much shorter antennal horns than $A$. gochnatiae, the last abdominal segment of both species exhibits a different number of dorsal spines (compare Fig. $8 \mathrm{C}$ with Fig. 9A) and only in A. gochnatiae there are some bifid spines (Fig. 9B), whereas all are simple in the new species. Larva of Asphondylia

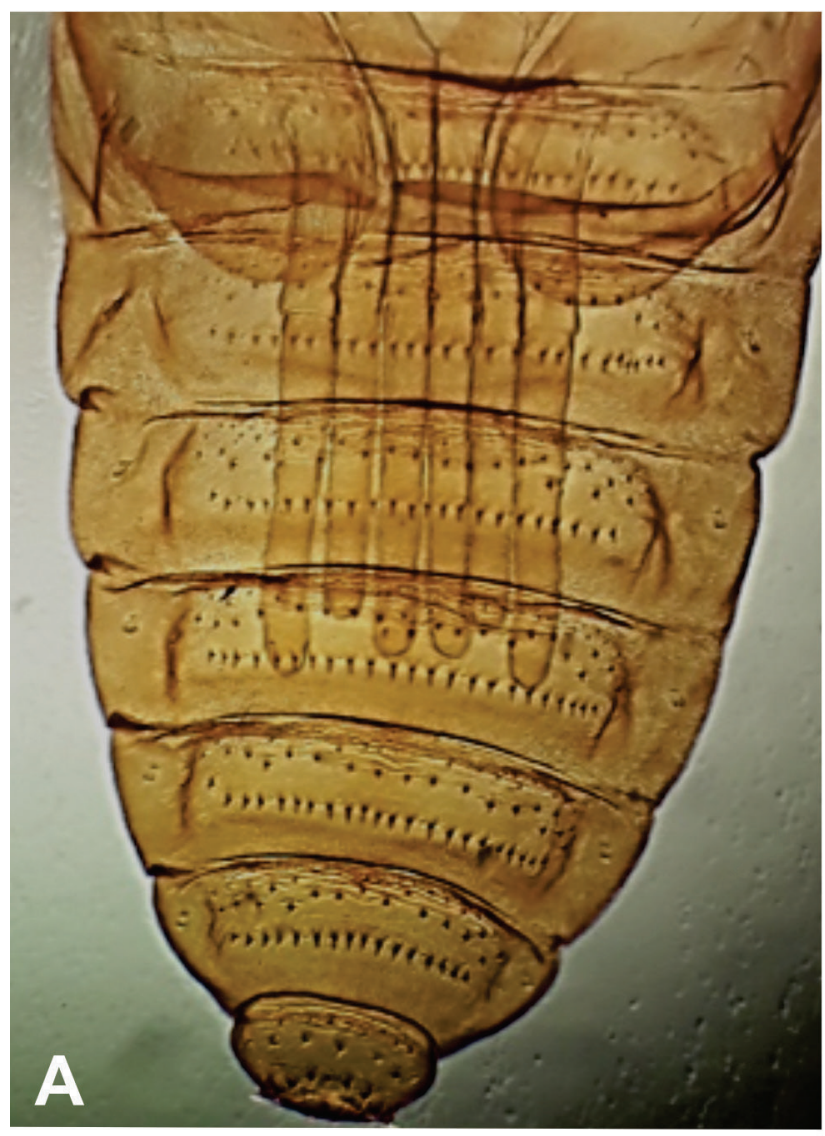

$0.50 \mathrm{~mm}$ ingaiensis has the inner teeth shorter than the outer ones, while in A. gochnatiae outer and inner teeth are similar in length.

Material examined of Asphondylia gochnatiae: 3 pupal exuviae, Minas Gerais: Luz, X.2005, E.O. Silveira col., MNRJ.

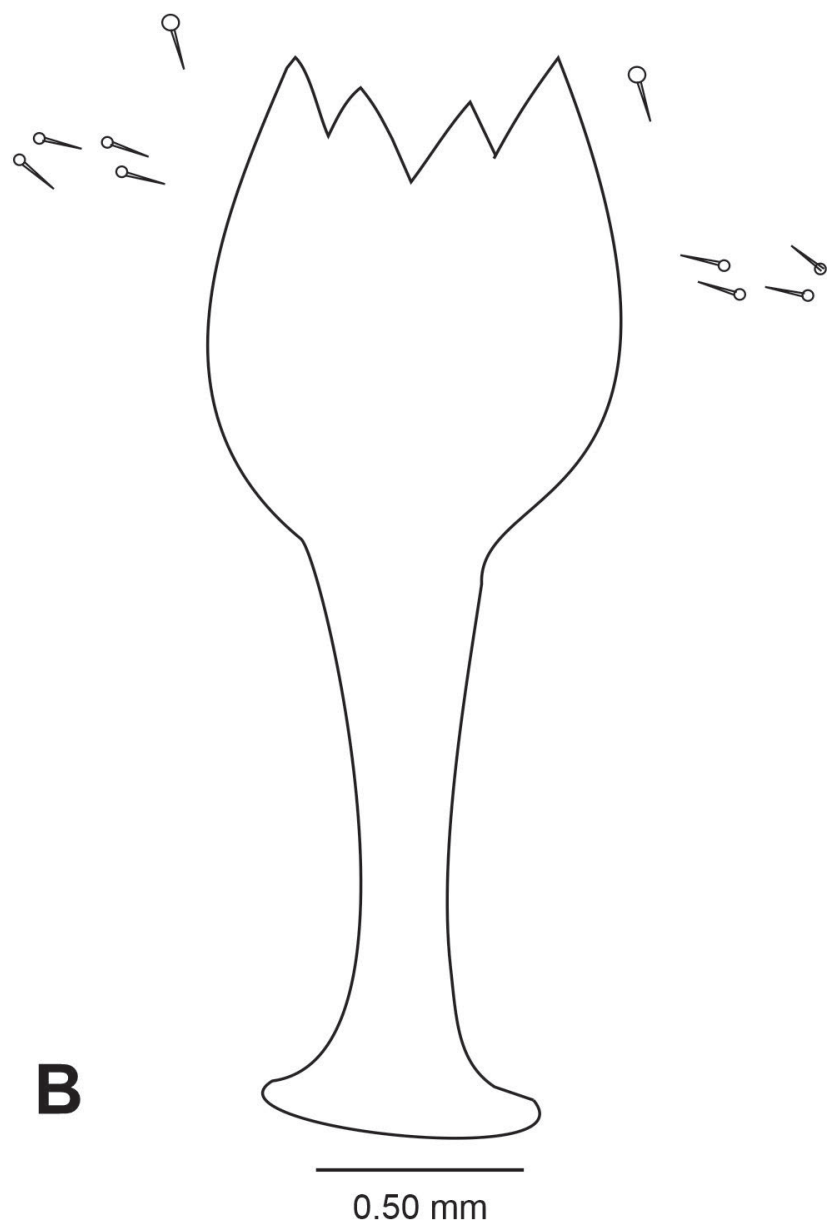

Figure 8. Asphondylia ingaiensis sp. nov. (A-B) Pupa, (A) Abdomen, dorsal view, (B) Larval spatula and associated papillae, ventral view.

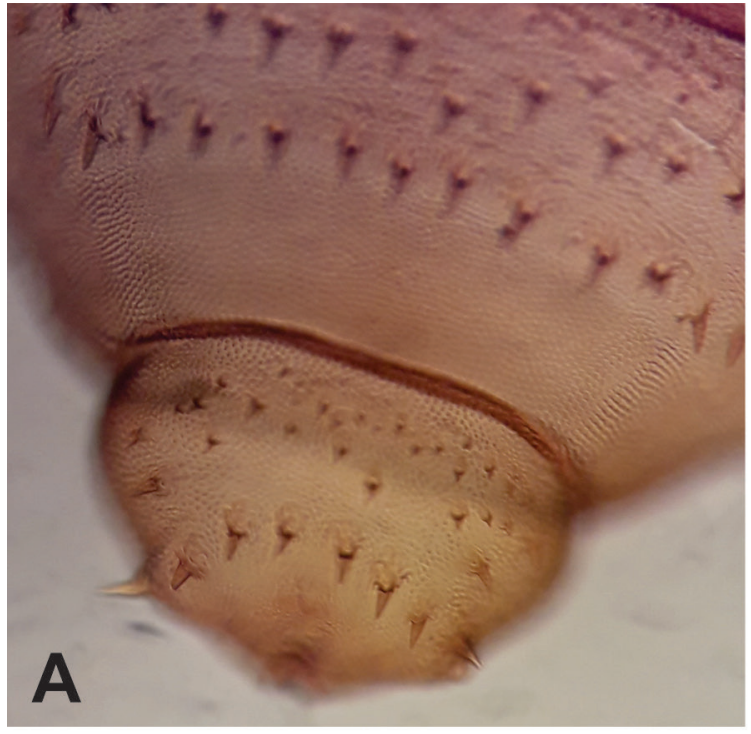

$0.43 \mathrm{~mm}$

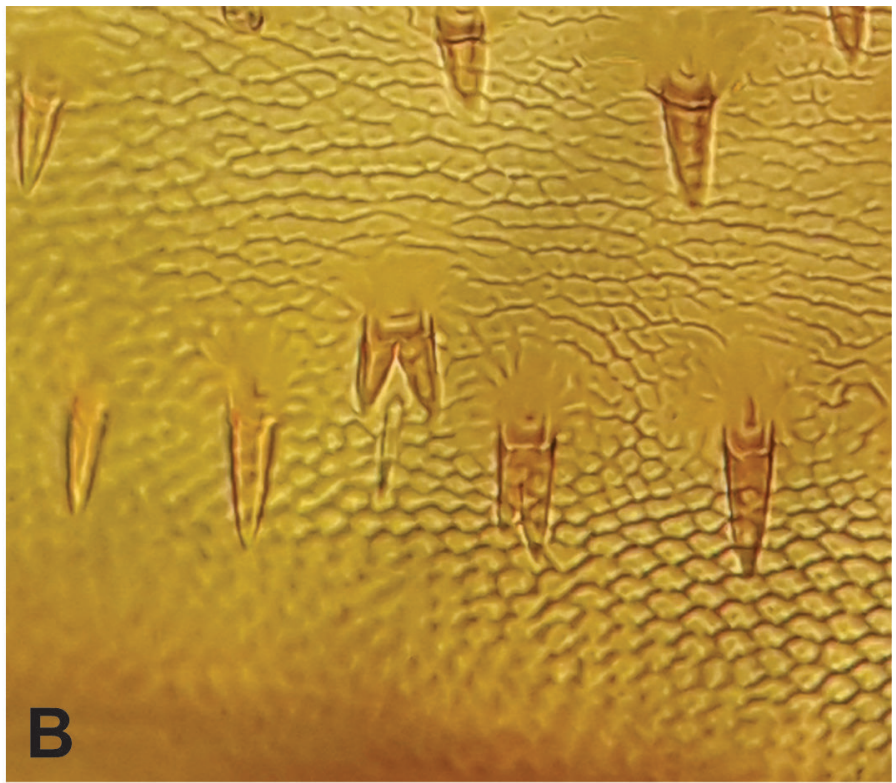

Figure 9. Asphondylia gochnatiae Maia, 2008, pupa. (A) Last abdominal segment, dorsal view, (B) Abdominal dorsal spines, dorsal view. 


\section{Asphondylia rufae Maia, sp. nov. (Figs. 10-15)}

Diagnosis: Male hypoproct acute apically, slightly bilobed; ovipositor with needle part 1.2-1.3 X length $7^{\text {th }}$ sternite, $7^{\text {th }}$ sternite with anterior and posterior margins more sclerotized mesally, pupa: antennal horn with apical part $0.8 \mathrm{X}$ length basal part, upper frontal horn bifid, $0.5 \mathrm{X}$ length antennal horn, lower frontal horn tridentate, $0.2 \mathrm{X}$ length antennal horn, $8^{\text {th }}$ abdominal segment with 6-17 dorsal spines in the posterior row.

Male: Body length: $3.50 \mathrm{~mm}(\mathrm{~N}=1)$. Head: 0.45-0.50 mm long, $0.50 \mathrm{~mm}$ wide $(\mathrm{N}=2)$, eye facets hexagonal, closely appressed (Fig. 10A); antennae: flagellomeres 1 and 2 not fused (Fig. 10B), scape truncated conical, setose, 0.12-0.15 mm long, $0.05 \mathrm{~mm}$ wide $(\mathrm{N}=4)$, pedicel globose, setose, $0.06 \mathrm{~mm}$ long, $0.05 \mathrm{~mm}$ wide $(\mathrm{N}=4)$, $1^{\text {st }}-12^{\text {th }}$ flagellomeres cylindrical, all 0.05-0.06 $\mathrm{mm}$ wide, circumfila longitudinally sinuous, equally spread along segments (Fig. 10C), $1^{\text {st }}$ flagellomere $0.21-0.26 \mathrm{~mm}$ long $(\mathrm{N}=5), 2^{\text {nd }}$ flagellomere $0.21-0.23 \mathrm{~mm}$ long $(\mathrm{N}=5)$, $3^{\text {rd }}-9^{\text {th }}$ flagellomeres $0.20-0.21 \mathrm{~mm}$ long $(\mathrm{N}=5), 10^{\text {th }}-12^{\text {th }}$ flagellomeres $0.18 \mathrm{~mm}$ long $(\mathrm{N}=3)$ (Fig. 10D), proportion flagellomere neck-node: 1:11; frons with 21 setae $(\mathrm{N}=2)$; mouth parts: labrum long-attenuate; hypopharynx of the same shape of labrum, with long lateral setulae, anteriorly directed; labella elongate and convex, 0.08-0.06 $\mathrm{mm}$ long, 0.06-0.04 $\mathrm{mm}$ wide $(\mathrm{N}=3)$, with lateral and mesal setae; palpus $0.26 \mathrm{~mm}$ long $(\mathrm{N}=2)$ : $1^{\text {st }}$ segment globoid, $0.03 \mathrm{~mm}$ long, $0.03 \mathrm{~mm}$ wide, $2^{\text {nd }}$ segment cylindrical, $0.07 \mathrm{~mm}$ long, $0.04 \mathrm{~mm}$ wide, $3^{\text {rd }}$ segment cylindrical. $0.17 \mathrm{~mm}$ long, $0.05 \mathrm{~mm}$ wide $(\mathrm{N}=2)$, all segments with setae. Thorax: scutum with two dors- ocentral rows of setae, setae more abundant anteriorly, and one irregular lateral row of setae on each side, scales intermixed; scutellum with scattered setae; anepimeron and anepisternum setose; remaining pleural sclerites bare; legs: tarsal claws curved beyond midlength, isomorphic, empodium as long as claws (Fig. 11A); first tarsomere of each leg with an apical hook-like projection $0.03 \mathrm{~mm}$ long ( $\mathrm{N}=14$ ) (Fig. 11B); wing: length $2.80 \mathrm{~mm}$ $(\mathrm{N}=1)$ (Fig. 11C). Abdomen (Fig. 11D): trichoid sensilla not visible; $1^{\text {st }} 7^{\text {th }}$ tergites sclerotized, rectangular with a posterior row of setae, few lateral setae, and mostly covered elsewhere with scales, 8th tergite bare, narrow, mesally constricted; $2^{\text {nd }}-8^{\text {th }}$ sternites more sclerotized than tergites, rectangular; $2^{\text {nd }}-6^{\text {th }}$ sternites with a posterior row of setae, several setae at midlength, few lateral setae, and mostly covered elsewhere with scales; $7^{\text {th }}$ sternites with a posterior row of setae, several mesal setae, few lateral setae, $8^{\text {th }}$ sternite entirely covered with setae, more abundant posteriorly, and mostly covered elsewhere with scales. Terminalia (Fig. 12A): gonocoxite short and stout, $0.18 \mathrm{~mm}$ long $(\mathrm{N}=1)$, gonostylus spherical, 0.07-0.08 mm long, 0.055-0.06 mm wide $(\mathrm{N}=3)$, teeth $0.01 \mathrm{~mm}$ long, $0.03 \mathrm{~mm}$ wide $(\mathrm{N}=2)$, hypoproct acute apically and slightly bilobed.

Female: Body length: $4.65 \mathrm{~mm}(\mathrm{~N}=1)$. Head: 0.50-0.65 mm long, 0.5-0.65 mm wide $(\mathrm{N}=4)$, antennae: scape $0.15 \mathrm{~mm}$ long $(\mathrm{N}=5)$, pedicel 0.06-0.07 $\mathrm{mm}$ long $(\mathrm{N}=7), 1^{\text {st }}-11^{\text {th }}$ flagellomeres cylindrical, $12^{\text {th }}$ spheroid, all $0.05 \mathrm{~mm}$ wide, circumfila comprising two longitudinal bands connected sub basally and apically by two transverse bands (Fig. 12B), $1^{\text {st }}$ flagellomere $0.26-0.30 \mathrm{~mm}$ long $(\mathrm{N}=8), 2^{\text {nd }}$ flagellomere $0.21-0.23 \mathrm{~mm}(\mathrm{~N}=8), 3^{\text {rd }}$ flagellomere $0.21-0.23 \mathrm{~mm}(\mathrm{~N}=8), 4^{\text {th }}$ and $5^{\text {th }}$ flagel-
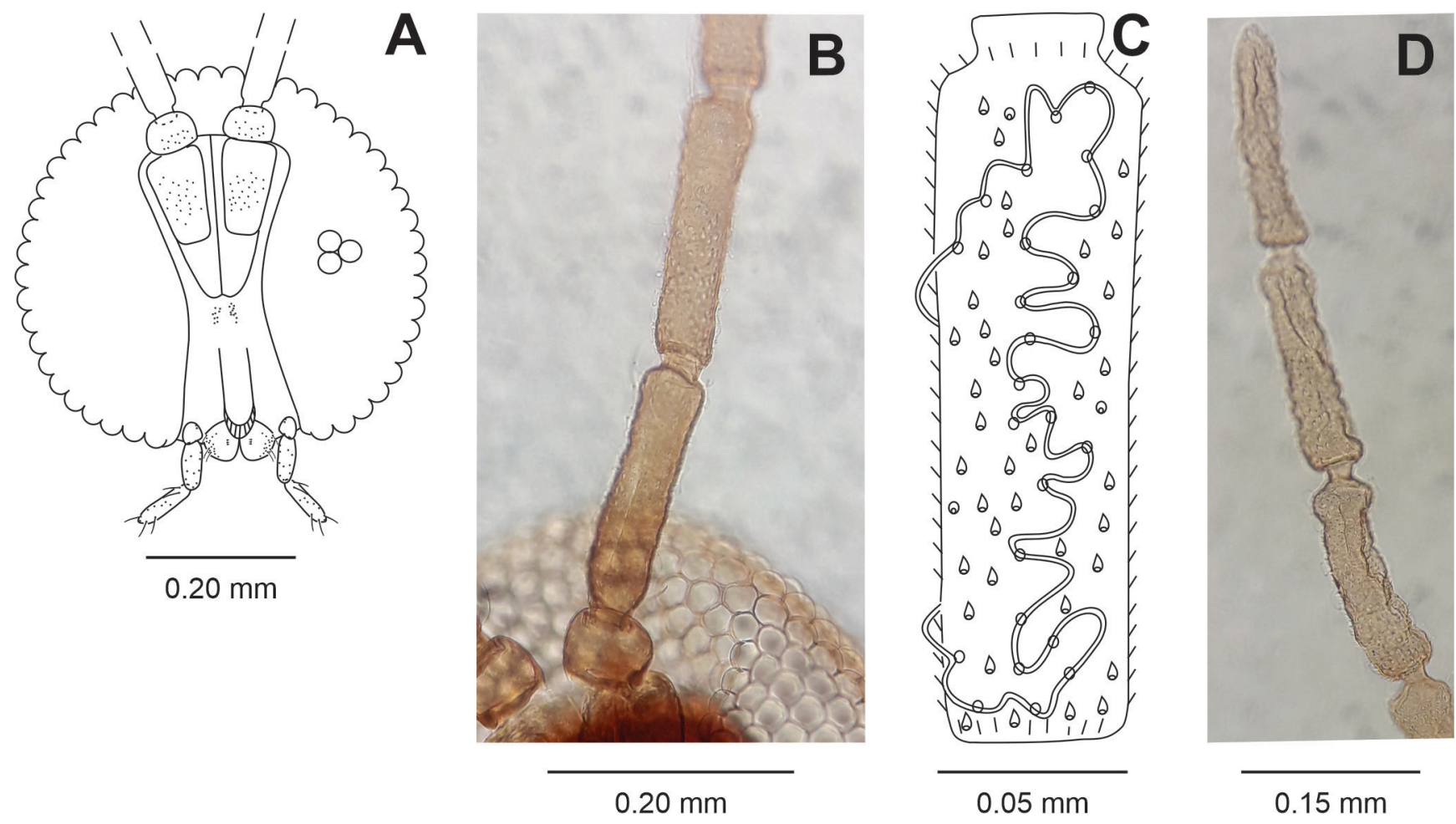

Figure 10. Asphondylia rufae sp. nov., male. (A) Head, ventral view, (B) $1^{\text {st }}$ and $2^{\text {nd }}$ flagellomeres, (C) $2^{\text {nd }}$ flagellomere, (D) $10^{\text {th }}-12^{\text {th }}$ flagellomeres. 

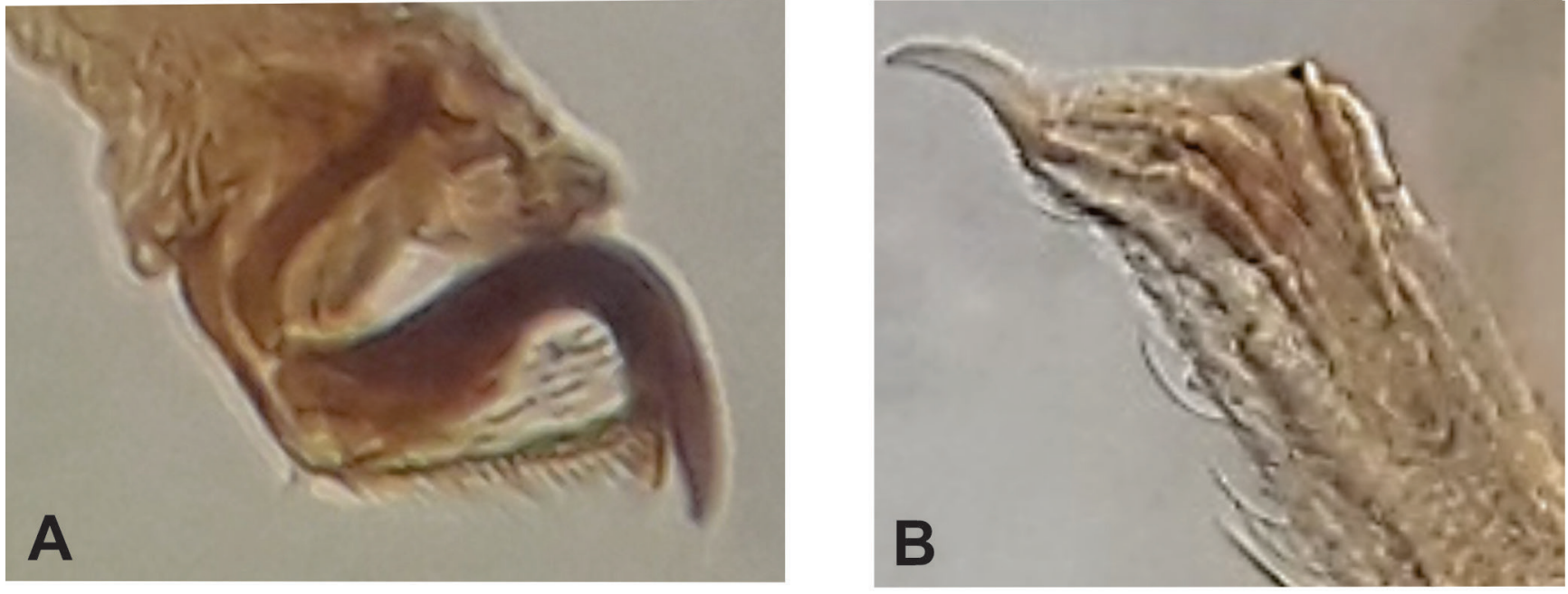

\section{$0.04 \mathrm{~mm}$}

$0.07 \mathrm{~mm}$

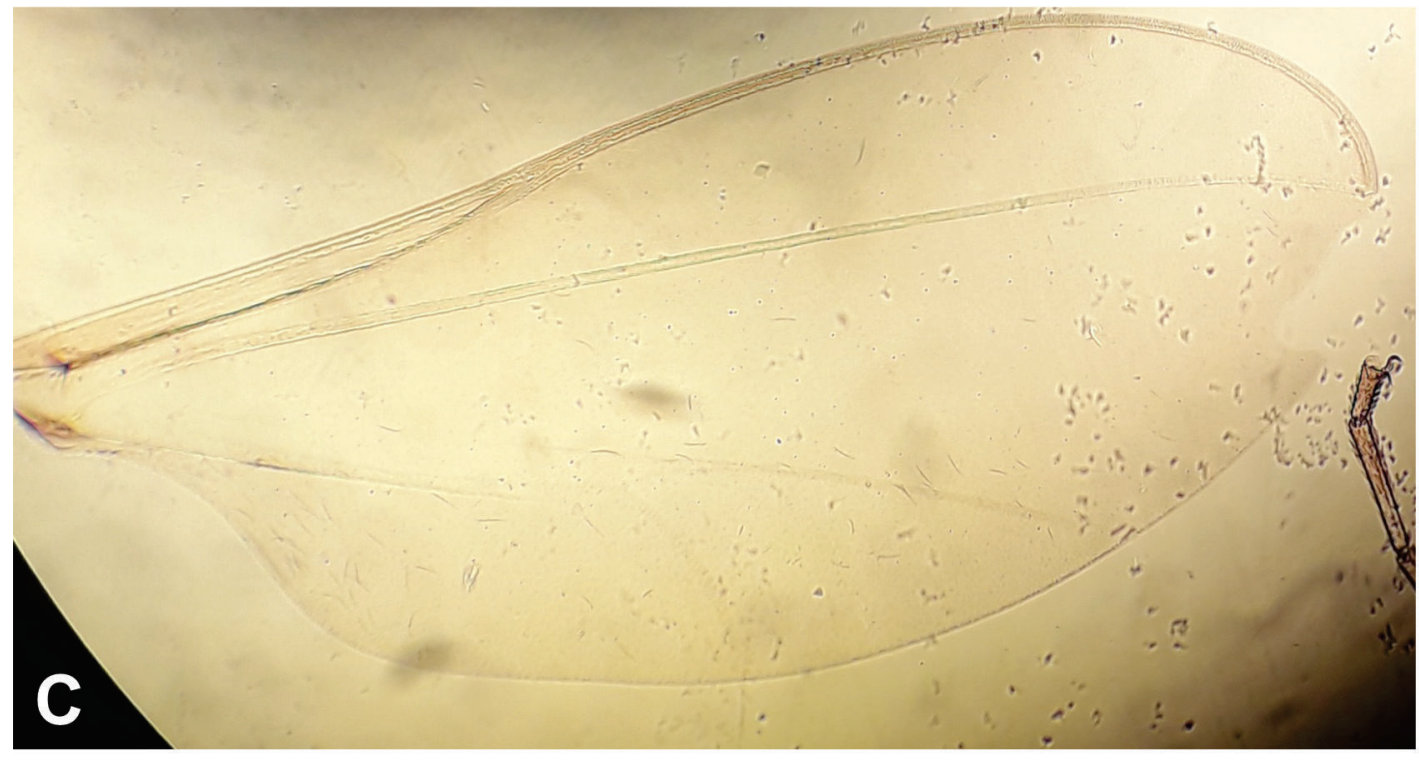

$1.40 \mathrm{~mm}$

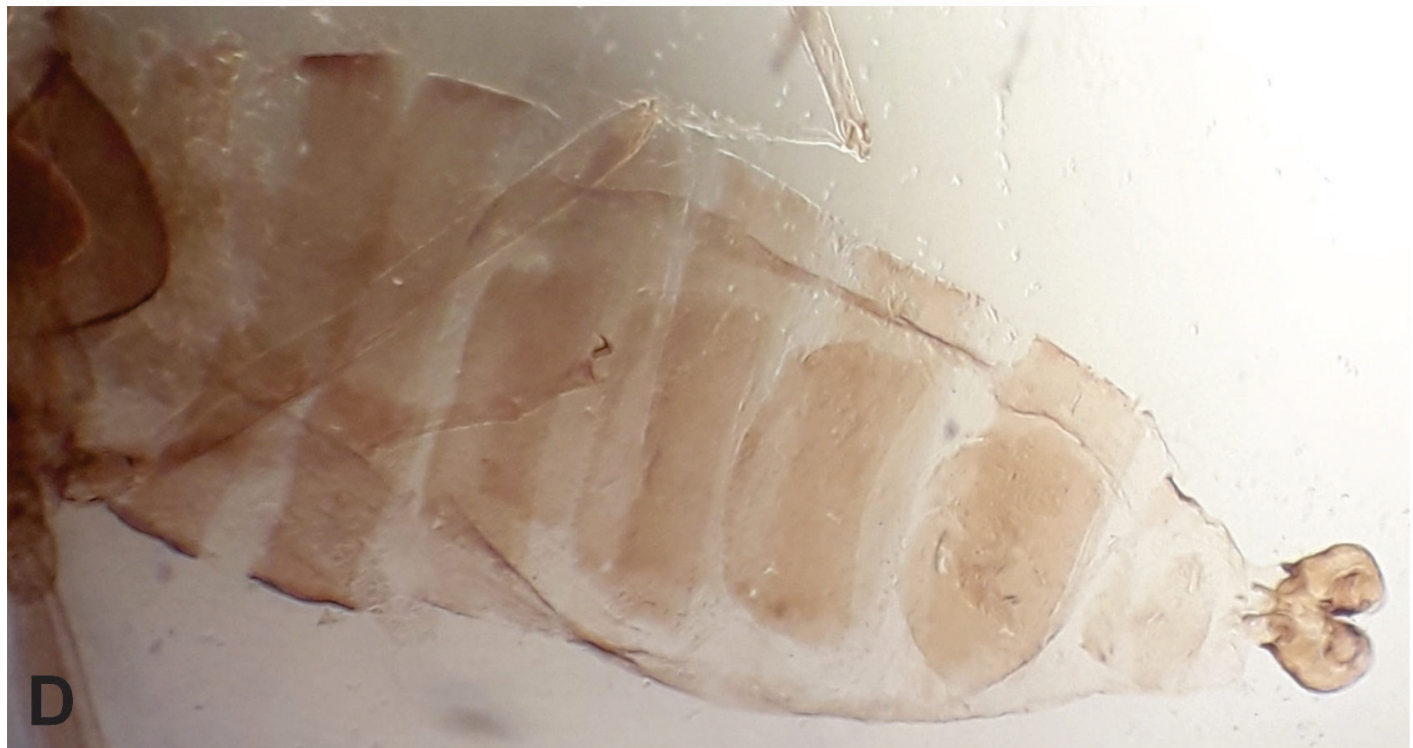

$0.50 \mathrm{~mm}$

Figure 11. Asphondylia rufae sp. nov., male. (A) Midleg, tarsal claw and empodium, lateral view (B) Hindleg, first tarsomere, lateral view, (C) Wing, (D) Abdomen, ventral view. 

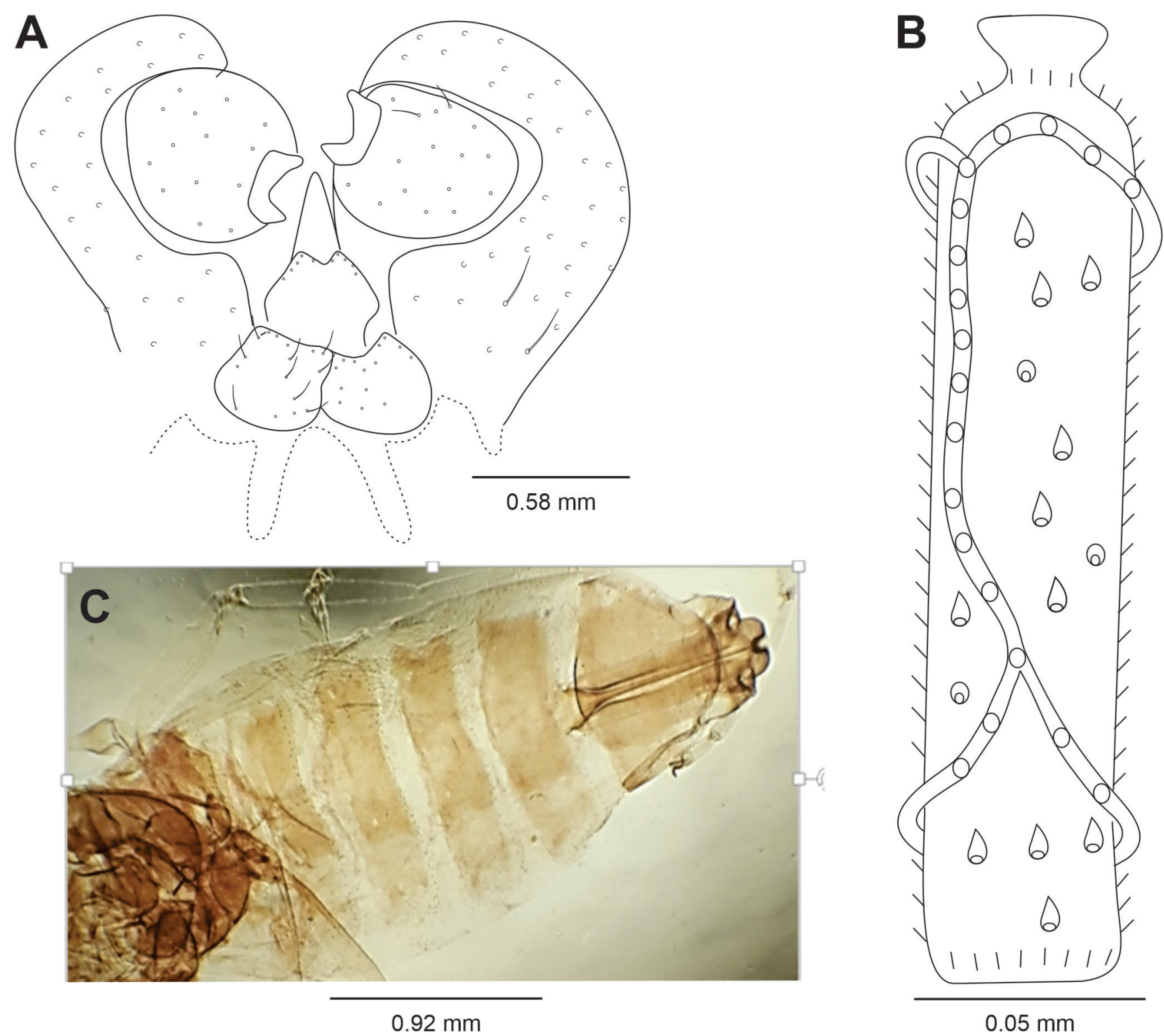

Figure 12. Asphondylia rufae sp. nov. (A) Male terminalia, dorsal view, (B-C) Female, (B) $4^{\text {th }}$ flagellomere, (C) Abdomen, ventral view.

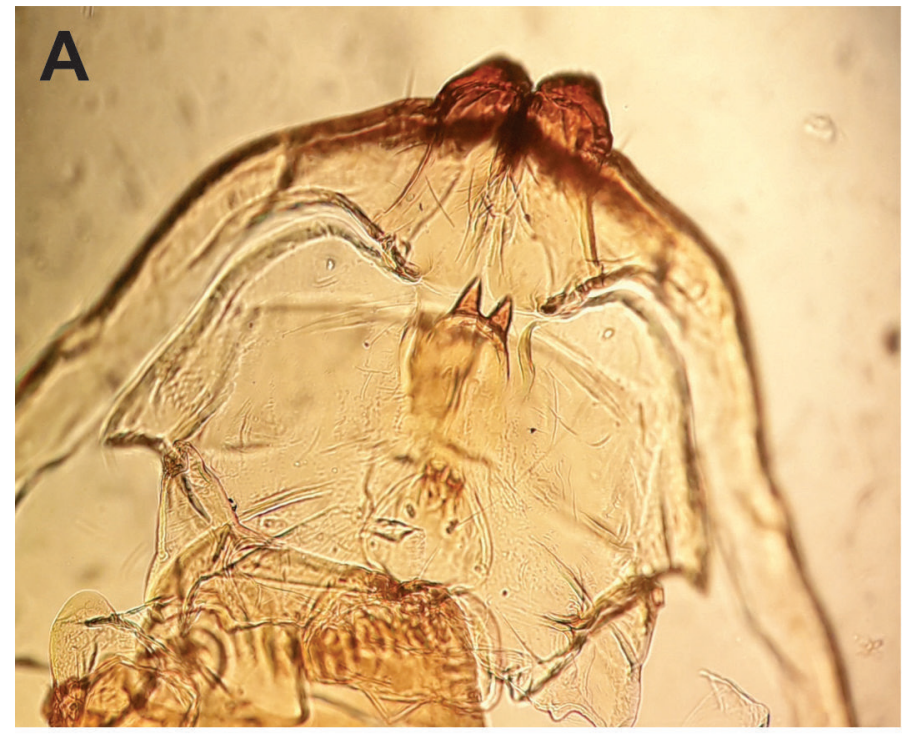

$0.20 \mathrm{~mm}$

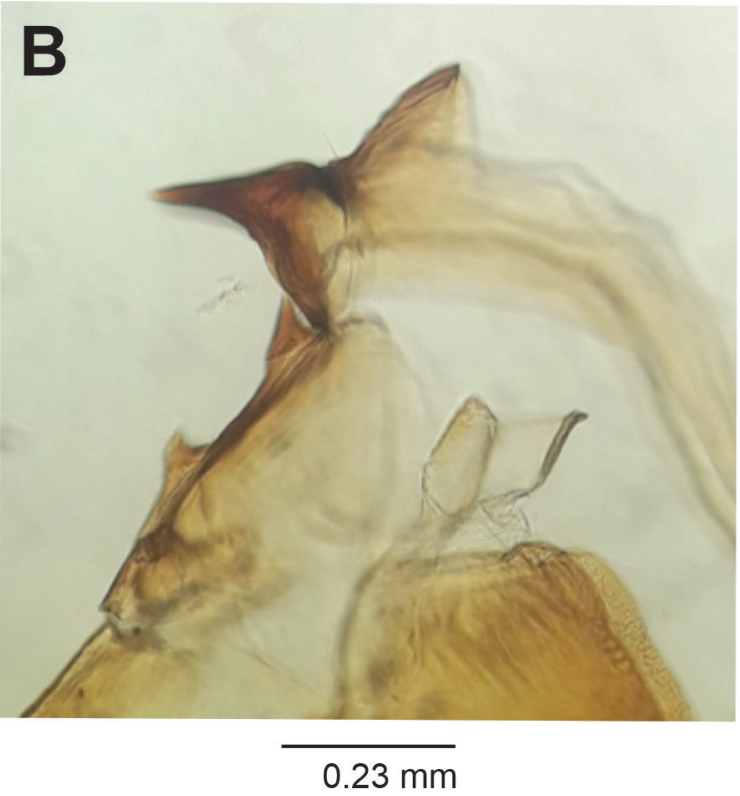

$0.23 \mathrm{~mm}$

Figure 13. Asphondylia rufae sp. nov., pupa. (A, B) Pupa head, ventral view, (A) ventral view, (B) lateral view. 


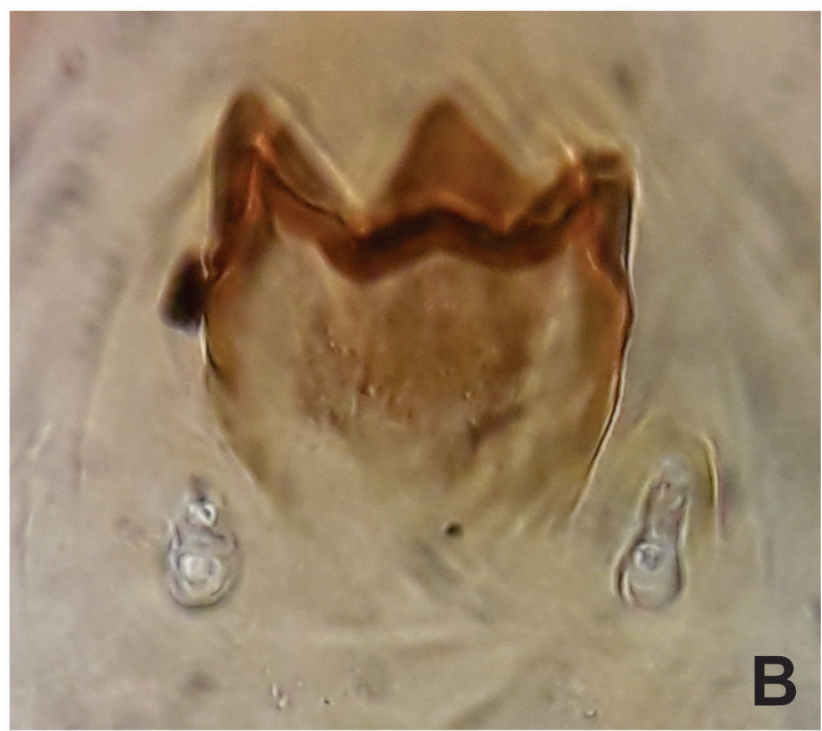

$0.06 \mathrm{~mm}$

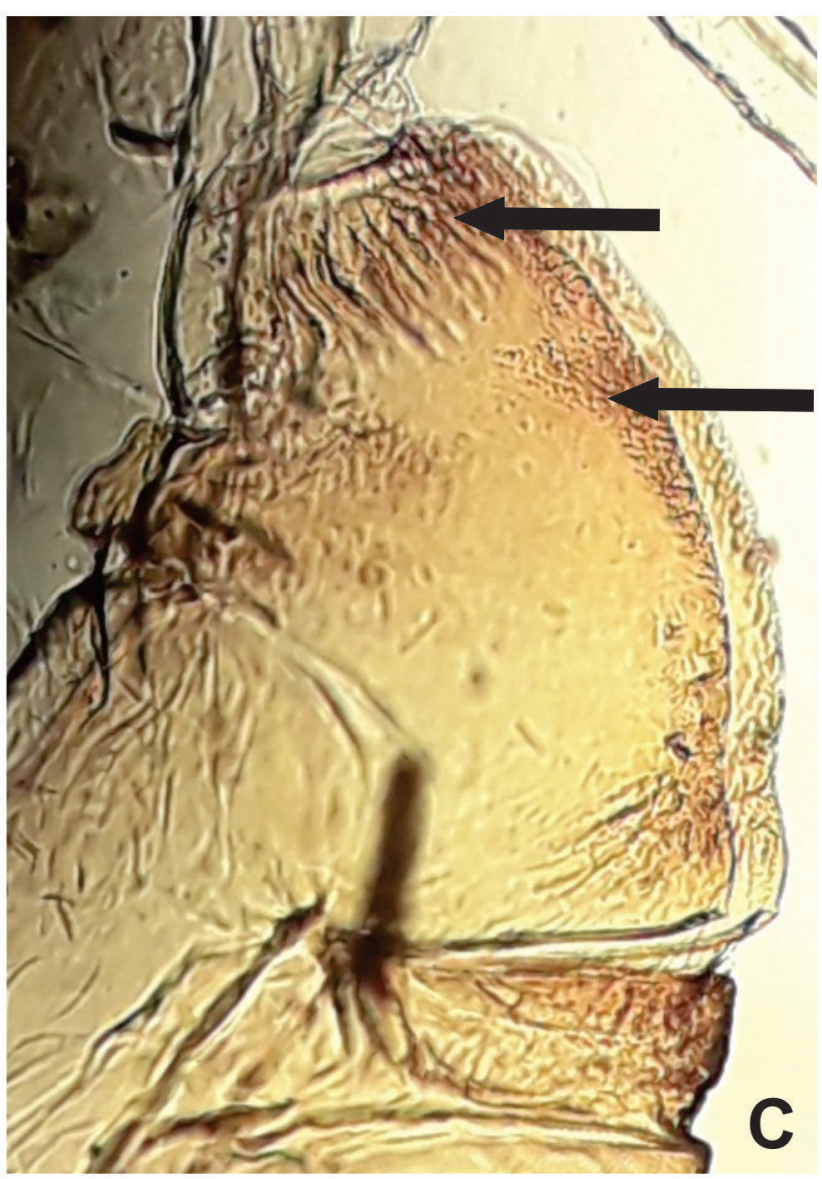

$0.30 \mathrm{~mm}$

Figure 14. Asphondylia rufae sp. nov., pupa. (A) Upper facial horn, (B) Lower facial horn, (C) Thorax integument, lateral view, (D) Last abdominal segments, dorsal view. 
lomeres $0.21-0.22 \mathrm{~mm}(\mathrm{~N}=5), 6^{\text {th }}$ and $7^{\text {th }}$ flagellomere $0.18-0.21 \mathrm{~mm}(\mathrm{~N}=4), 8^{\text {th }}$ flagellomeres $0.18-0.20 \mathrm{~mm}$ $(\mathrm{N}=3) ; 9^{\text {th }}$ flagellomere $0.14-0.17 \mathrm{~mm}(\mathrm{~N}=3), 10^{\text {th }}$ flagellomere $0.08-0.15 \mathrm{~mm}$ long $(\mathrm{N}=3), 11^{\text {th }}$ flagellomere $0.06-0.10 \mathrm{~mm}(\mathrm{~N}=3), 12^{\text {th }}$ flagellomere $0.05 \mathrm{~mm}$ long $(\mathrm{N}=3)$; mouthparts: labellum 0.08-0.15 mm long, $0.05 \mathrm{~mm}$ wide at midlength $(\mathrm{N}=3)$, palpus $0.28 \mathrm{~mm}$ long $(\mathrm{N}=2)$ : $1^{\text {st }}$ segment globose $0.03 \mathrm{~mm}$ long and $0.025 \mathrm{~mm}$ wide $(\mathrm{N}=2), 2^{\text {nd }}$ segment cylindrical $0.08 \mathrm{~mm}$ long, $0.03 \mathrm{~mm}$ wide at midlength $(\mathrm{N}=2), 3^{\text {rd }}$ segment claviform, $0.17 \mathrm{~mm}$ long and $0.02 \mathrm{~mm}$ wide at midlength $(\mathrm{N}=2)$. Thorax: wing length: $3.30-3.80 \mathrm{~mm}(\mathrm{~N}=10)$; legs: tarsal claws more robust than in male, apical projection of first tarsomere with 0.035-0.05 mm long ( $=4$ ). Abdomen (Fig. 12C): trichoid sensillae not visible, $1^{\text {st }} 7^{\text {th }}$ tergites sclerotized, rectangular with a posterior row of setae, few lateral setae and mostly covered elsewhere with scales, $8^{\text {th }}$ tergite with distal margin with lobes $0.16-0.17 \mathrm{~mm}$ long $(\mathrm{N}=3), 2^{\text {nd }}-5^{\text {th }}$ sternites as in male, $6^{\text {th }}$ sternite with a single posterior row of setae, several setae and midlength, lateral setae, mostly covered elsewhere with scales; $7^{\text {th }}$ sternite with anterior margin and posterior margins more sclerotized mesally, 0.61-0.74 mm long, 2.03-2.05 X length sternite 6 $(\mathrm{N}=5)$, setose (except basally), and mostly covered elsewhere with scales; sternite 8 not sclerotized; ovipositor: needle part 0.78-0.90 mm long, 1.22-1.28 X length sternite $7(n=5)$. Other characters as in male.

Pupa: Color: brownish. Body length: 3.90-4.45 mm $(\mathrm{N}=8)$. Head (Figs. 13A, B): antennal horn 0.24-0.33 mm long $(\mathrm{N}=4)$, triangular, with inner margin serrated; apical seta $0.05 \mathrm{~mm}$ long ( $\mathrm{N}=3$ ); upper facial horn bifid, 0.13-0.14 mm long ( $=4)$, triangular (Fig. 14A); lower facial horn tridentate, 0.05-0.06 mm long $(\mathrm{N}=4)$, triangular (Fig. 14B); two pairs of lower facial papillae: one pair setose, the other bare; three pairs of lateral facial papillae: one pair setose, two bare; upper cephalic margin thickened laterally. Thorax: integument wrinkly (Fig. 14C), prothoracic spiracle $0.14-0.20 \mathrm{~mm}$ long, setiform, curved near distal third $(\mathrm{N}=3)$. Abdomen: segments 2-8 with transverse rows of crescent dorsal spines (Fig. 14D); posterior row with 14-20 spines in the $2^{\text {nd }}$ segment, 16-21 in the $3^{\text {rd }}, 17-19$ in the $4^{\text {th }}, 16-17$ in the $5^{\text {th }}, 15-16$ in the $6^{\text {th }}$, 12 in the $7^{\text {th }}$ and $6-7$ in the $8^{\text {th }}(\mathrm{N}=3)$ (Fig. 14D).

\section{Larva: Unknown.}

Gall: On stem, fusiform, brown, glabrous (Fig. 15).

Material examined: Holotype male, BRAZIL, Minas Gerais, Dores do Indaiá, IX.2009, L.A. Oliveira col. (MNRJ).

Paratypes: Same data as holotype, 6 males, 10 females, and 5 pupal exuviae (MNRJ).

Etymology: The name "rufae" is the genitive of the specific epithet of the host plant.

Remarks: Asphondylia rufae induces galls on Bauhinia rufa (Fabaceae). Four other gall midge species were pre-

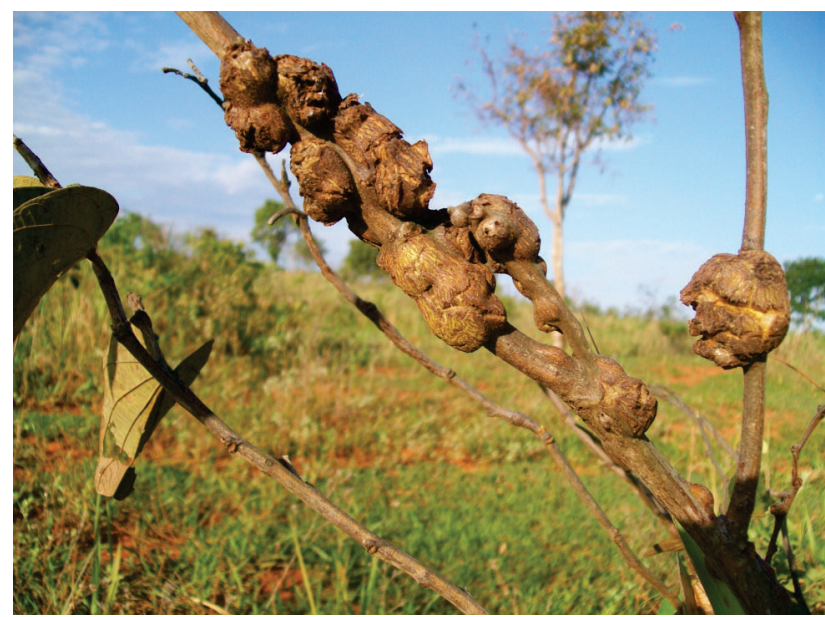

Figure 15. Asphondylia rufae sp. nov., stem gall on Bauhinia rufa (Fabaceae), general aspect.
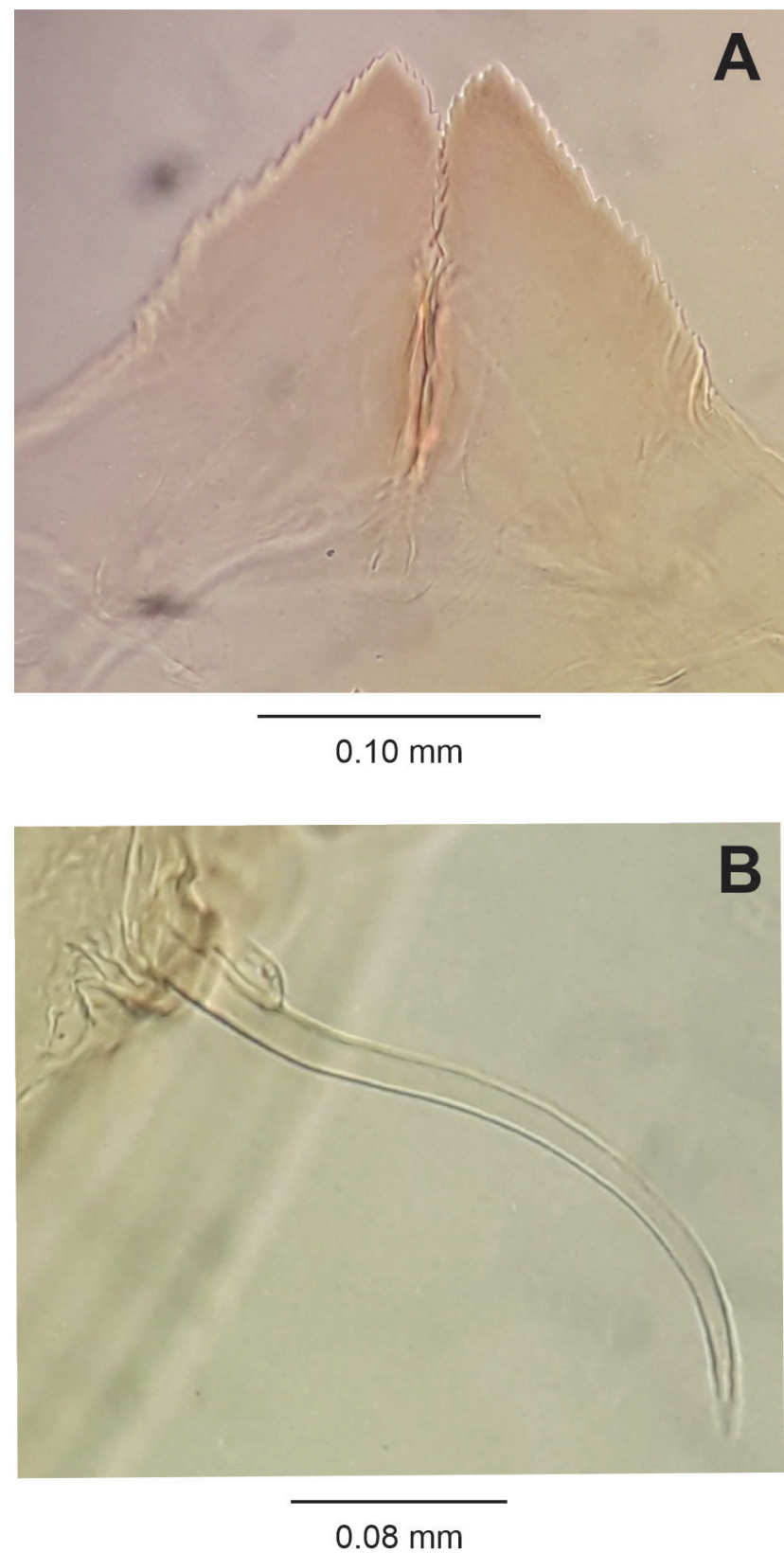

Figure 16. Asphondylia microcapillata Maia, 2005, pupa. 63. Antennal horn, 64. Prothoracic spiracle. 
viously recorded on this plant genus: Asphondylia microcapillata, Schizomyia macrocapillata (both on Bauhinia brevipes), S. barreirensis on B. cupulata and S. tuiuiu on $B$. holophylla. Adults of the new species differs from A. microcapillata in the number of frontal setae (21 in the former and about 40 in latter), shape of male hypoproct (more acute apically in A. rufae than in A. microcapillata), and needle part length of ovipositor (as long as $7^{\text {th }}$ sternite in the new species and about $2.0 \mathrm{X}$ length $7^{\text {th }}$ sterni- te in A. microcapillata). Pupa differs in the antennal horn shape (with serrated outer margin only in A. microcapillata, Fig. 16A), upper facial horn (bifid in both species, but much longer in $A$. rufae), and lower frontal spines (present in the new species and absent in A. microcapillata). The prothoracic spiracle is as long as antennal horn in A. microcapillata and shorter than antennal horn in A. rufa. Besides, it has rounded apex in the new species and pointed apex in A. microcapillata (Fig. 16B).
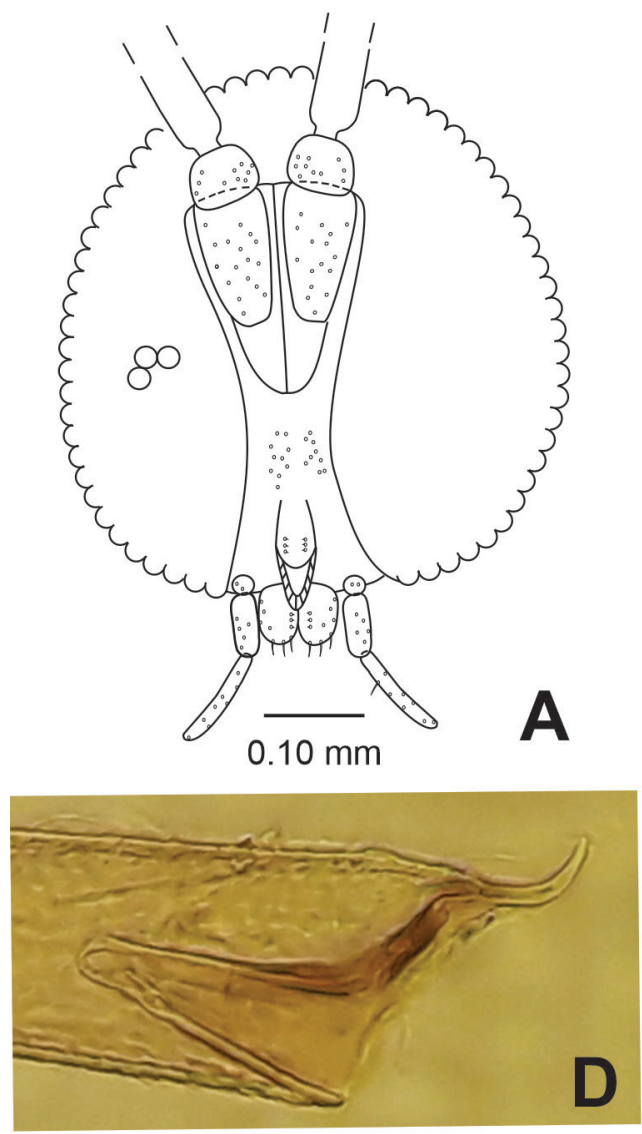

$0.07 \mathrm{~mm}$

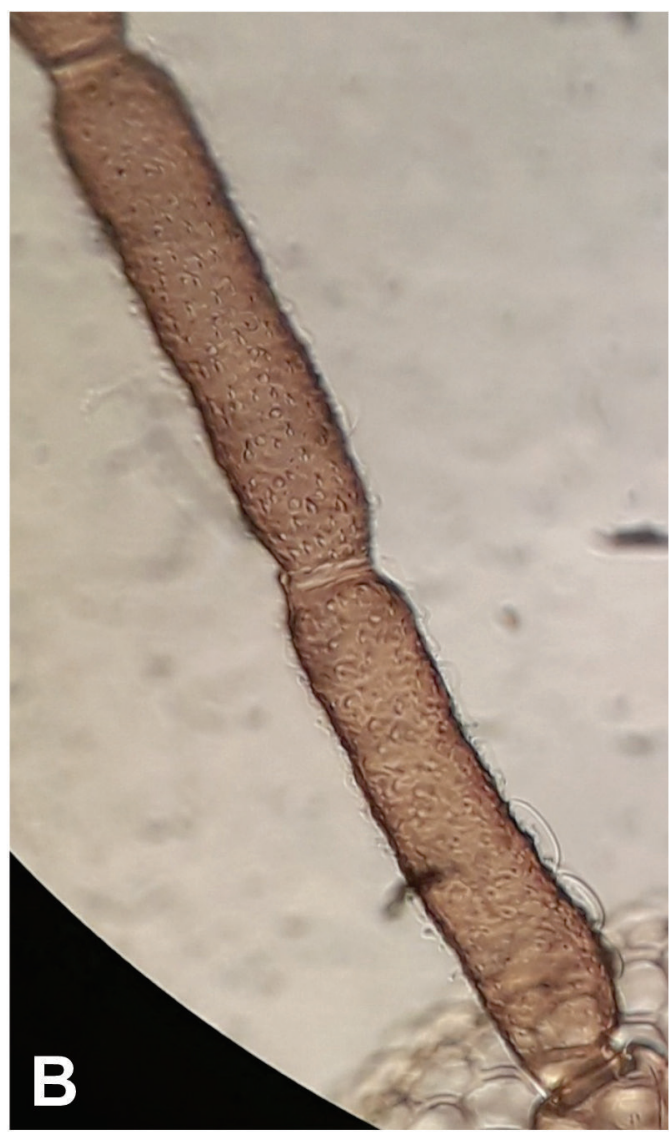

$0.10 \mathrm{~mm}$

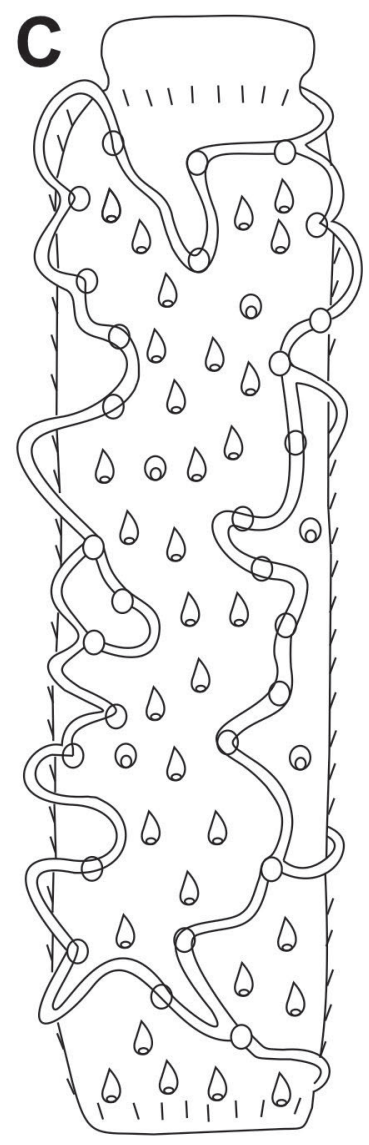

$0.05 \mathrm{~mm}$

Figure 17. Asphondylia zeyheriae sp. nov., male. (A) Head, ventral view, (B) $1^{\text {st }}$ and $2^{\text {nd }}$ flagellomeres, (C) $3^{\text {rd }}$ flagellomere, (D) Midleg, $1^{\text {st }}$ tarsomere, lateral view.

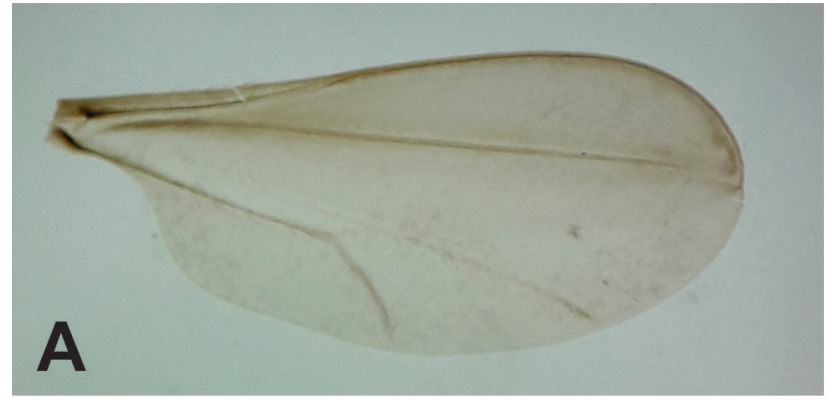

$0.40 \mathrm{~mm}$

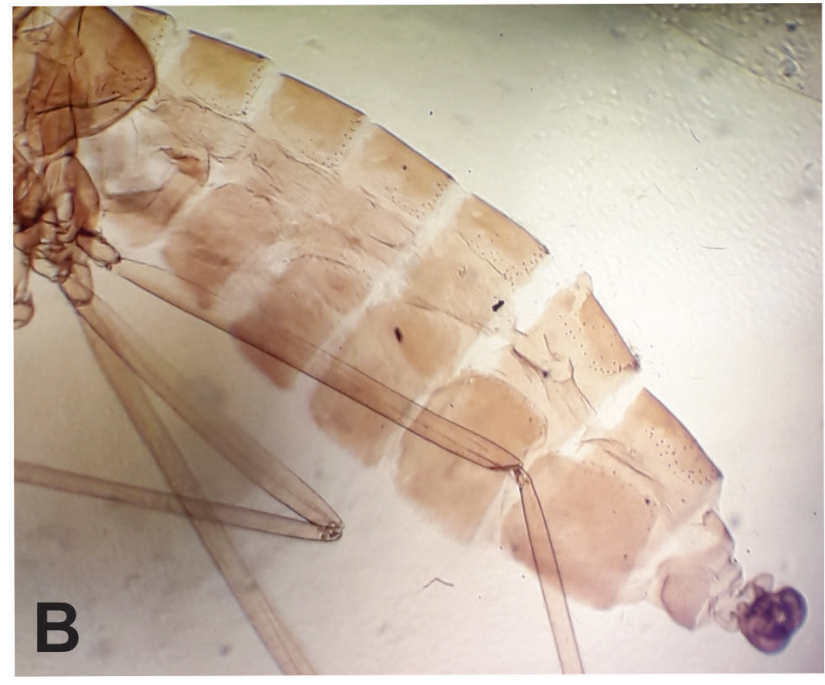

$0.50 \mathrm{~mm}$

Figure 18. Asphondylia zeyheriae sp. nov., male. (A) Wing, (B) Abdomen, lateral view. 
Material Examined of Asphondylia microcapillata: 7 pupal exuviae: Minas Gerais: Três Marias, XII.2004, E.C. Pereira leg., MNRJ.

\section{Asphondylia zeyheriae Maia, sp. nov. (Figs. 17-23)}

Diagnosis: Male hypoproct rounded apically, deeply bilobed; ovipositor with needle part about 3.0-4.0 X length $7^{\text {th }}$ sternite, pupa: antennal horn apical part $2.5 \mathrm{X}$ length basal part, upper frontal horn simple, $0.4 \mathrm{X}$ length antennal horn, lower facial horn tridentate, $0.2 \mathrm{X}$ length antennal horn, face sclerotized between upper and lower facial horns, $8^{\text {th }}$ abdominal segment with 5 dorsal spines in the posterior row.

Male: Body length: $4.20 \mathrm{~mm}(\mathrm{~N}=1)$. Head (Fig. 17A): $0.55 \mathrm{~mm}$ long, $0.50 \mathrm{~mm}$ wide $(\mathrm{N}=1)$, eye facets hexagonal,

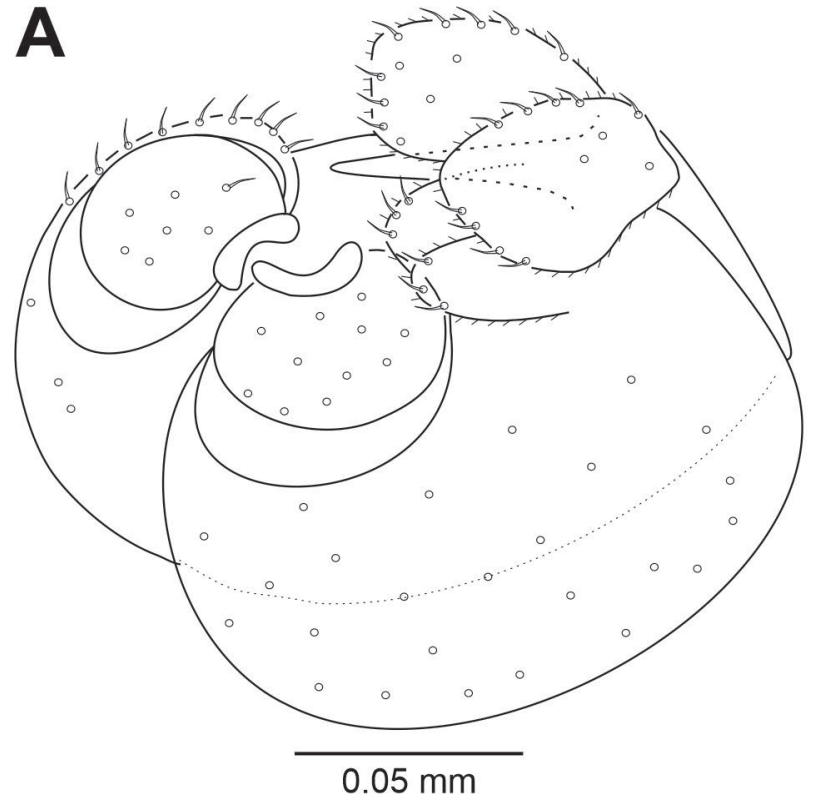

closely appressed; antennae: flagellomeres 1 and 2 not fused (Fig. 17B), scape truncated conical, setose, $0.14 \mathrm{~mm}$ long, $0.05 \mathrm{~mm}$ wide $(\mathrm{N}=1)$, pedicel globose, setose, $0.05 \mathrm{~mm}$ long, $0.05 \mathrm{~mm}$ wide $(\mathrm{N}=1), 1^{\text {st }}-10^{\text {th }}$ flagellomeres cylindrical (other flagellomeres missing), all $0.05 \mathrm{~mm}$ wide, circumfila longitudinally wavy, dense, anastomosing, equally spread along segments (Fig. 17C), $1^{\text {st }}$ and $2^{\text {nd }}$ flagellomeres $0.22 \mathrm{~mm}$ long $(\mathrm{N}=1), 3^{\text {rd }}-6^{\text {th }}$ flagellomeres $0.20 \mathrm{~mm}$ long $(\mathrm{N}=1), 7^{\text {th }}-8^{\text {th }}$ flagellomeres $0.17 \mathrm{~mm}$ long $(\mathrm{N}=1)$, proportion flagellomere neck-node: $1: 11$; frons with 18 setae $(\mathrm{N}=1)$; mouth parts: labrum long-attenuate; hypopharynx of the same shape of labrum, with long lateral setulae anteriorly directed; labella elongate and convex, $0.05 \mathrm{~mm}$ long, $0.035 \mathrm{~mm}$ wide $(\mathrm{N}=1)$, with lateral and mesal setae; palpus about $0.26 \mathrm{~mm}$ long $(\mathrm{N}=1)$ : $1^{\text {st }}$ segment globoid, $0.02 \mathrm{~mm}$ long, $0.02 \mathrm{~mm}$ wide, $2^{\text {nd }}$ segment $0.065 \mathrm{~mm}$ long, $0.025 \mathrm{~mm}$ wide, $3^{\text {rd }}$ segment $0.18 \mathrm{~mm}$ long, $0.15 \mathrm{~mm}$ wide $(\mathrm{N}=1)$, all segments with setae.

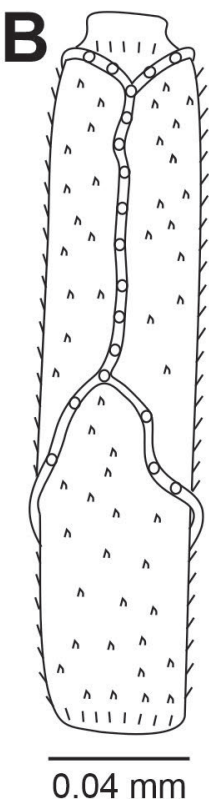

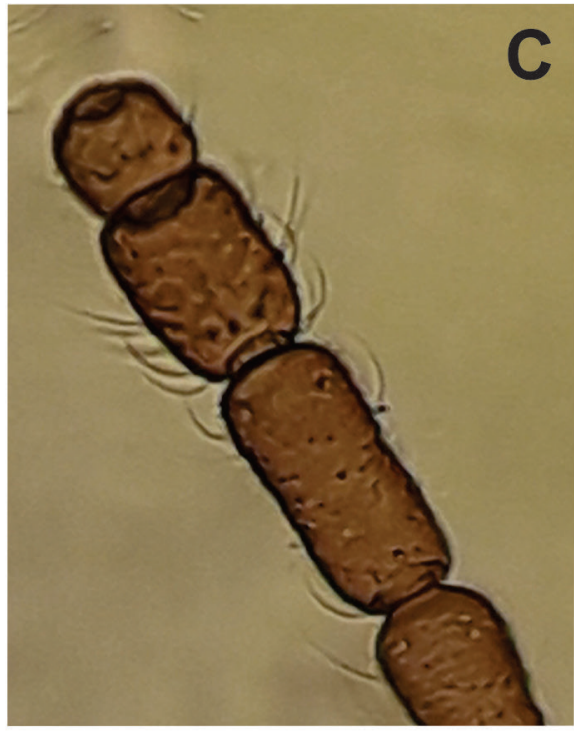

$0.13 \mathrm{~mm}$

Figure 19. Asphondylia zeyheriae sp. nov., (A) Male terminalia, dorsal view, (B-C) Female antenna, (B) $6^{\text {th }}$ flagellomere, (C) $10^{\text {th }}-12^{\text {th }}$ flagellomeres.

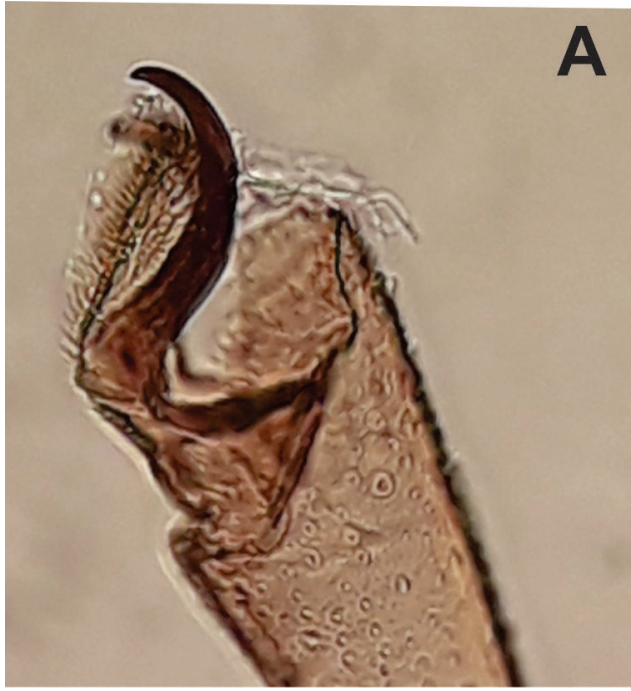

$0.07 \mathrm{~mm}$

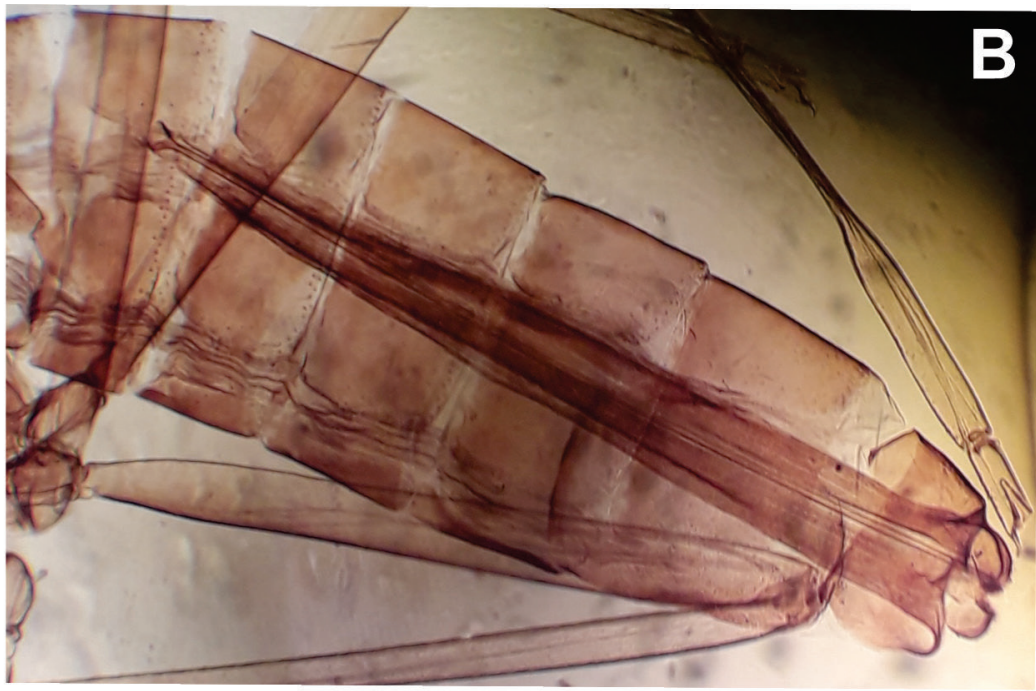

$0.80 \mathrm{~mm}$

Figure 20. Asphondylia zeyheriae sp. nov., female. (A) Foreleg, tarsal claw and empodium, lateral view, (B) Abdomen, dorsal view. 


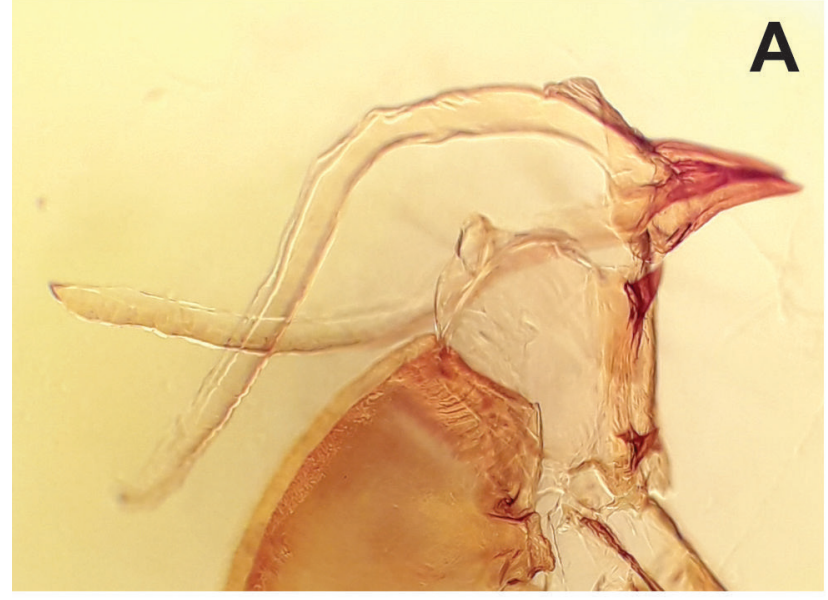

$$
0.45 \mathrm{~mm}
$$
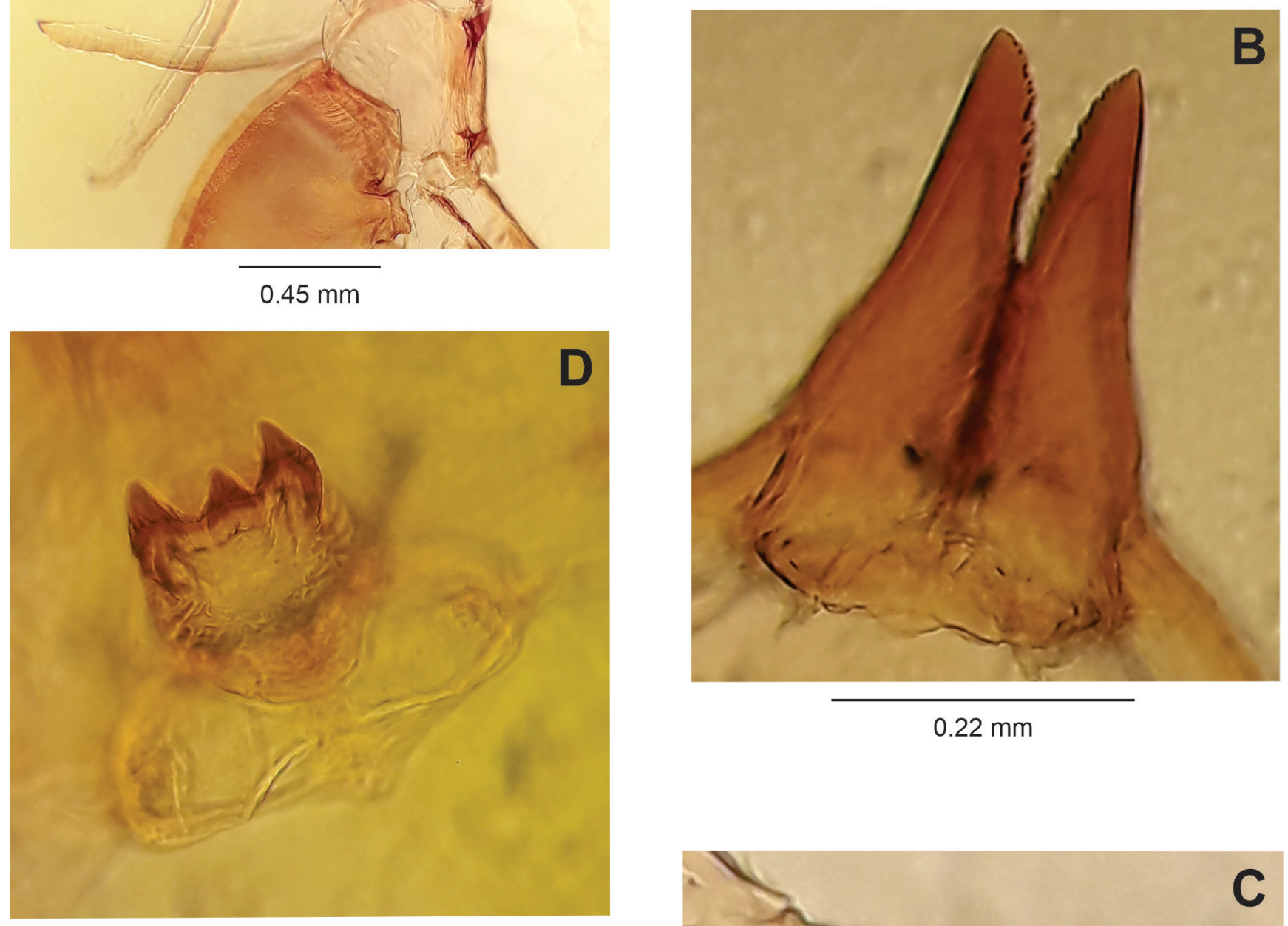

$0.22 \mathrm{~mm}$
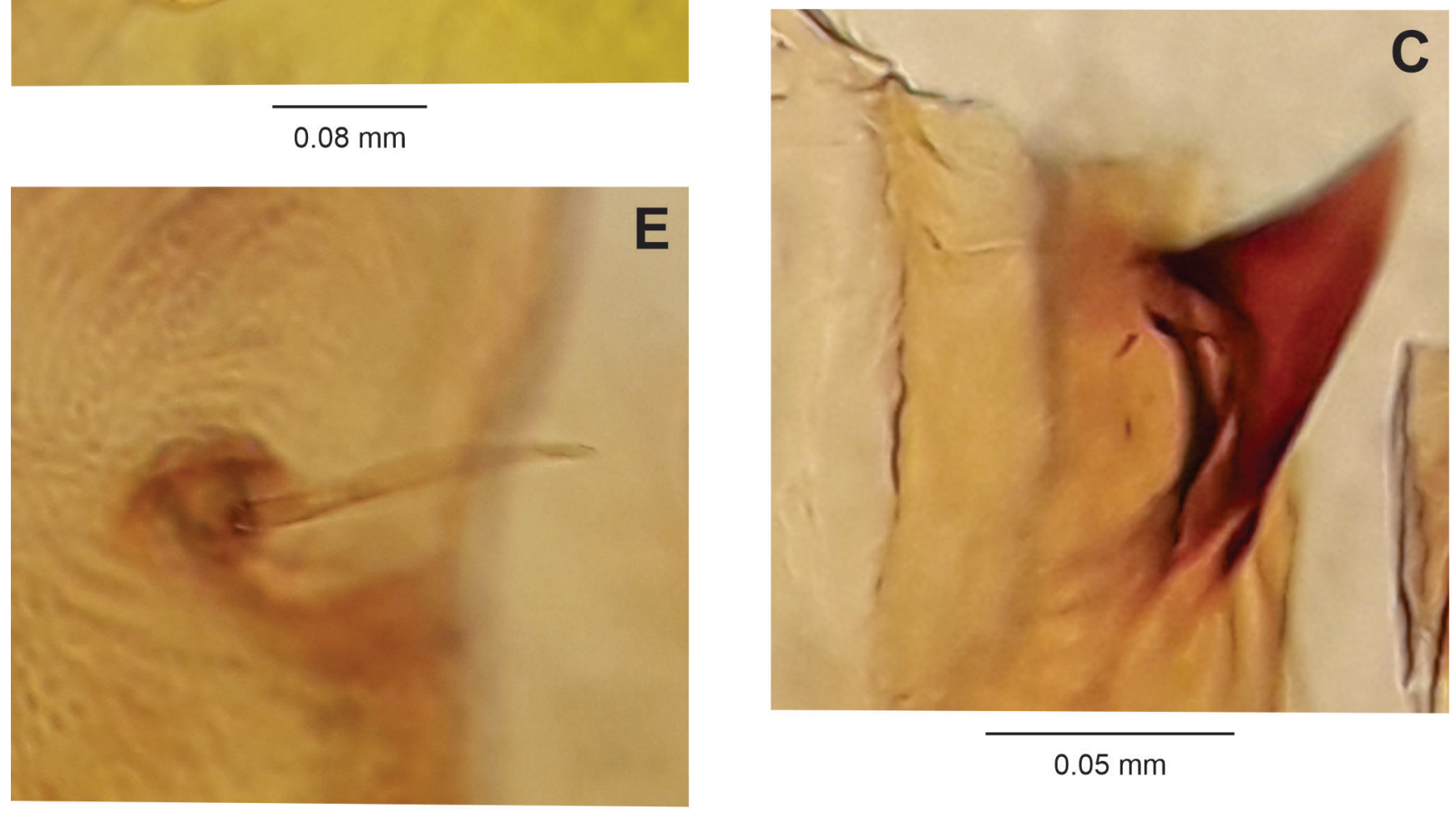

$0.18 \mathrm{~mm}$

Figure 21. Asphondyliazeyheriae sp. nov., pupa. (A) Head, lateral view, (B) Antennal horn, (C) Upper facial horn, (D) Lower facial horn, (E) Prothoracic spiracle, dorsal view. 
Thorax: scutum with two dorsocentral rows of setae, setae more abundant anteriorly and posteriorly, one lateral row of setae on each side of scutum, extending from base to distal margin, scales intermixed; scutellum with scattered setae; anepimeron setose, anepisternum setose; remaining pleural sclerites bare; legs: first tarsomere of each leg with an apical hook-like projection 0.04-0.05 mm long $(\mathrm{N}=2)$ (Fig. 17D), all legs broken; wing: length $2.80 \mathrm{~mm}$ $(\mathrm{N}=1)$ (Fig. 18A). Abdomen (Fig. 18B): trichoid sensilla not visible; $1^{\text {st }} 7^{\text {th }}$ tergites sclerotized, rectangular with a posterior row of setae, few lateral setae and mostly covered elsewhere with scales, $8^{\text {th }}$ tergite bare, narrow, mesally constricted; $2^{\text {nd }}-8^{\text {th }}$ sternites more sclerotized than tergites; $2^{\text {nd }}-6^{\text {th }}$ sternites rectangular with a posterior row of setae, several setae at midlength, few lateral setae, mostly covered elsewhere with scales; $6^{\text {th }}$ sternite with anterior and posterior margins slightly concave; $7^{\text {th }}$ sternite rectangular with a posterior row of setae, several mesal setae, few lateral setae, $8^{\text {th }}$ sternite rectangular, entirely covered with setae, more abundant posteriorly, and mostly covered elsewhere with scales. Terminalia (Fig. 19A): gonocoxite short and stout, $0.16-0.18 \mathrm{~mm}$ long, $0.10 \mathrm{~mm}$ wide $(\mathrm{N}=2)$, gonostylus spherical $0.05-0.06 \mathrm{~mm}$ long, $0.07 \mathrm{~mm}$ wide $(\mathrm{N}=2)$, teeth $0.01 \mathrm{~mm}$ long, $0.025 \mathrm{~mm}$ wide $(\mathrm{N}=2)$, hypoproct rounded apically, deeply bilobed.

Female: Body length: 4.40-6.50 mm ( $\mathrm{N}=3)$. Head: 0.65-0.70 mm long, $0.56-0.65 \mathrm{~mm}$ wide $(\mathrm{N}=2)$, antennae: scape $0.16 \mathrm{~mm}$ long $(\mathrm{N}=1)$, pedicel $0.06 \mathrm{~mm}$ long $(\mathrm{N}=1), 1^{\text {st }}-11^{\text {th }}$ flagellomeres cylindrical, all $0.05 \mathrm{~mm}$ wide, circumfila comprising two longitudinal bands connected sub basally and apically by two transverse bands

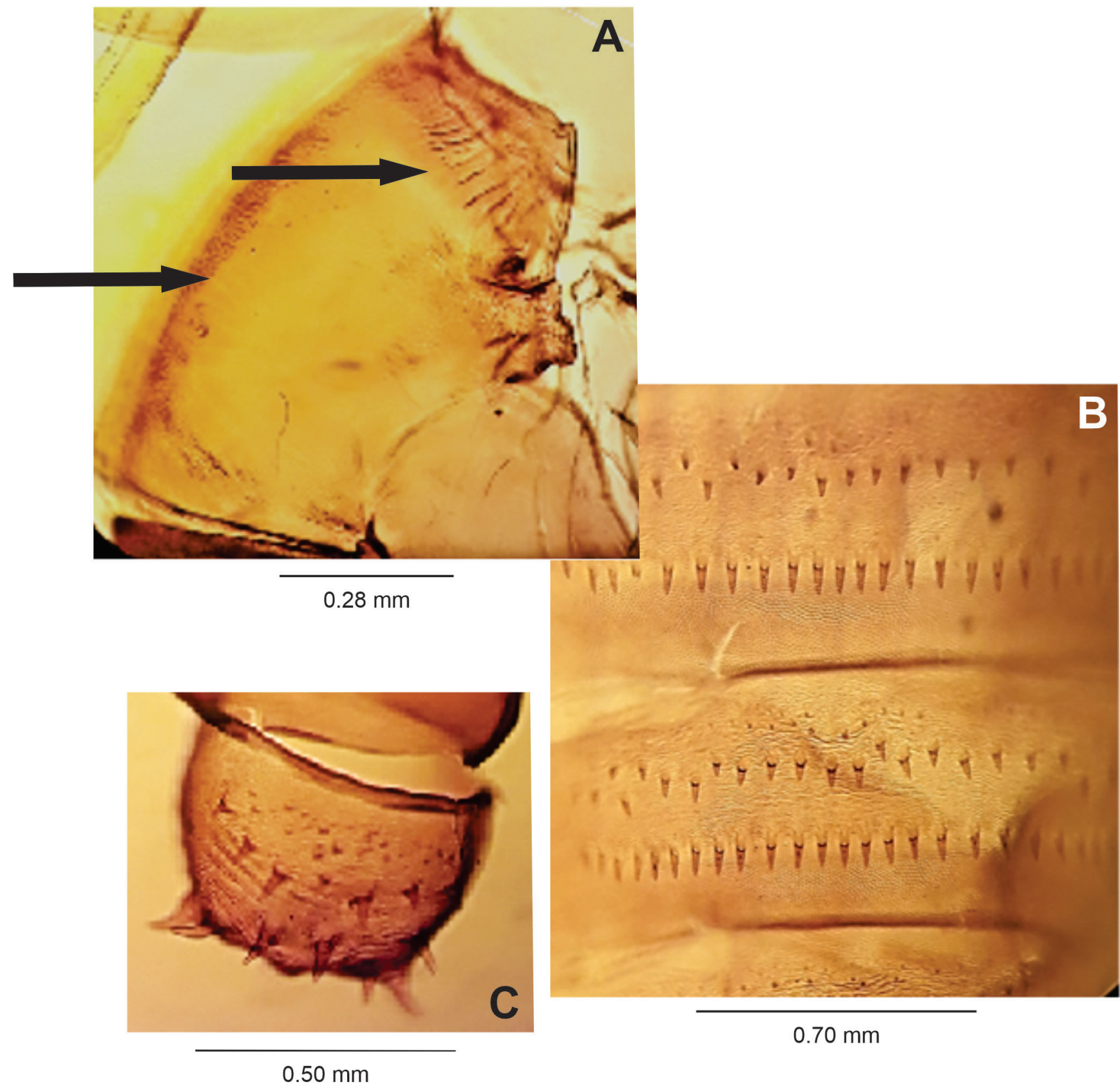

Figure 22. Asphondylia zeyheriae sp. nov., pupa. (A) Thorax integument, lateral view, (B) dorsal spines on $5^{\text {th }}-6^{\text {th }}$ abdominal segments, dorsal view, (C) Terminal segment, dorsal view. 
(Fig. 19B), $1^{\text {st }}$ flagellomere $0.30 \mathrm{~mm}$ long $(\mathrm{N}=1), 2^{\text {nd }}$ and $3^{\text {rd }}$ flagellomeres $0.25 \mathrm{~mm}$ long $(\mathrm{N}=1), 4^{\text {th }}$ and $5^{\text {th }}$ flagellomeres $0.22 \mathrm{~mm}$ long $(\mathrm{N}=1), 6^{\text {th }}$ and $7^{\text {th }}$ flagellomere $0.20 \mathrm{~mm}(\mathrm{~N}=1), 8^{\text {th }}$ flagellomeres $0.18 \mathrm{~mm}$ long $(\mathrm{N}=1)$; $9^{\text {th }}$ flagellomere $0.15 \mathrm{~mm}$ long $(\mathrm{N}=1), 10^{\text {th }}$ flagellomere $0.10 \mathrm{~mm}$ long $(\mathrm{N}=1), 11^{\text {th }}$ flagellomere $0.07 \mathrm{~mm}$ long $(\mathrm{N}=1), 12^{\text {th }}$ flagellomere $0.05 \mathrm{~mm}$ long $(\mathrm{N}=1)$ (Fig. 19C); mouthparts: palpus $0.24 \mathrm{~mm}$ long $(\mathrm{N}=2)$ : $1^{\text {st }}$ segment globose $0.02-0.03 \mathrm{~mm}$ long, $0.02 \mathrm{~mm}$ wide $(\mathrm{N}=2), 2^{\text {nd }}$ segment cylindrical $0.08 \mathrm{~mm}$ long, $0.03 \mathrm{~mm}$ wide at midlength $(\mathrm{N}=3), 3^{\text {rd }}$ segment cylindrical $0.13 \mathrm{~mm}$ long and 0.02-0.03 $\mathrm{mm}$ wide at midlength $(\mathrm{N}=3)$. Thorax: anepimeron setose; legs: tarsal claws curved beyond midlength, anisomorphic, less robust on foreleg than on mid and hindlegs, empodium as long as claws (Fig. 20A); apical hook-like projection of first tarsomere with 0.04-0.05 mm long $(\mathrm{N}=3)$; wing: length 3.30-4.10 $\mathrm{mm}(\mathrm{N}=4)$. Abdomen (Fig. 20B): trichoid sensillae not visible, $1^{\text {st }} 7^{\text {th }}$ tergites as in male, $8^{\text {th }}$ tergite with posterior margin with lobes $0.20 \mathrm{~mm}$ long $(\mathrm{N}=2), 2^{\text {nd }}-6^{\text {th }}$ sternites as in male, $7^{\text {th }}$ sternite with anterior and posterior margins more sclerotized mesally, $0.65 \mathrm{~mm}$ long, $2.41 \mathrm{X}$ length sternite $6(\mathrm{~N}=1)$, setose (except basally), and mostly covered elsewhere with scales; sternite 8 not sclerotized; ovipositor: needle part 2.10-2.62 mm long ( $\mathrm{N}=4), 3.23-4.00 \mathrm{X}$ length sternite 7 $(n=4)$. Other characters as in male.

Pupa: Color: brownish. Body length: 5.00-6.40 mm $(\mathrm{N}=2)$. Head (Fig. 21A): antennal horn $0.48 \mathrm{~mm}$ long $(\mathrm{N}=1)$, triangular, with inner margin serrated (Fig. 21B); apical seta $0.06 \mathrm{~mm}$ long $(\mathrm{N}=1)$; upper facial horn simple, 0.17-0.20 mm long ( $\mathrm{N}=2$ ), triangular (Fig. 21C); lower facial horn tridentate, $0.10 \mathrm{~mm}$ long $(\mathrm{N}=2)$, triangular (Fig. 21D); face sclerotized between upper and lower facial horns (Fig. 21A); two pairs of lower facial papillae: one pair setose, the other bare; three pairs of lateral facial papillae: one pair setose, two bare; upper cephalic margin thickened laterally. Thorax: prothoracic spira-

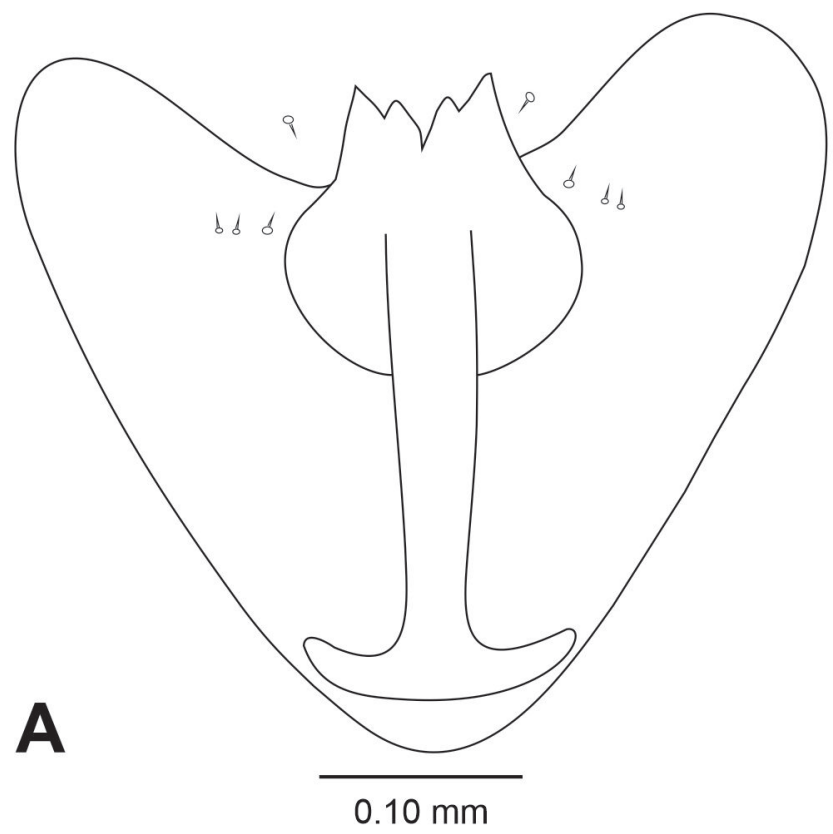

cle 0.11-0.13 mm long, setiform, slightly curved $(\mathrm{N}=3)$ (Fig. 21E), integument wrinkly (Fig. 22A). Abdomen: segments 2-8 with transverse rows of crescent dorsal spines (Fig. 22B); posterior row with 23 spines in the $2^{\text {nd }}$ segment, 21-24 in the $3^{\text {rd }}, 22-25$ in the $4^{\text {th }}, 21-24$ in the $5^{\text {th }}, 19-20$ in the $6^{\text {th }}, 10-14$ in the $7^{\text {th }}$ and 5 in the $8^{\text {th }}(N=2)$ (Fig. 22C).

Larva: Body length: $3.70-3.90 \mathrm{~mm}(\mathrm{~N}=1)$; cephalic head $0.30 \mathrm{~mm}$ long, $0.54 \mathrm{~mm}$ wide. Prothoracic spatula (Fig. 23A) 0.38-0.40 mm long $(\mathrm{N}=2)$, four-toothed, inner teeth shorter than outer, three setose lateral papillae on each side. Terminal segment with four setose papillae (Fig. 23B).

Gall: On flower bud. No other morphological features are available in the labels of the specimens.

Material examined: Holotype male, BRAZIL, Minas Gerais, Uberlândia, E.E. do Panga, III.2005, J.F. Andrade col. (MNRJ).

Paratypes: Same data as holotype, 1 male, 3 females, and 5 pupal exuviae; Minas Gerais, Serra do Cipó, 25.VI.2002, N. Bittencourt col., 2 larvae; VII.2002, same collector, 1 female and 2 pupal exuviae (MNRJ).

Etymology: The name "zeyheriae" is the genitive of the host plant genus.

Remarks: Asphondylia zeyheriae induces galls on Zeyheria montana Mart. (Bignoniaceae), a plant endemic to Brazil with records in the Amazon Forest, Atlantic Forest, Caatinga, and Cerrado (Lohmann, 2020). This is the first gall midge species reported on this plant.

Only one other congeneric species, Asphondylia godmaniae Möhn, 1959, from El Salvador, induces galls on Bignoniaceae. It is known only from larva and pupa (Möhn, 1959). The described stages of Asphondylia godmaniae and A. zeyheriae are morphologically very similar, but their lar-

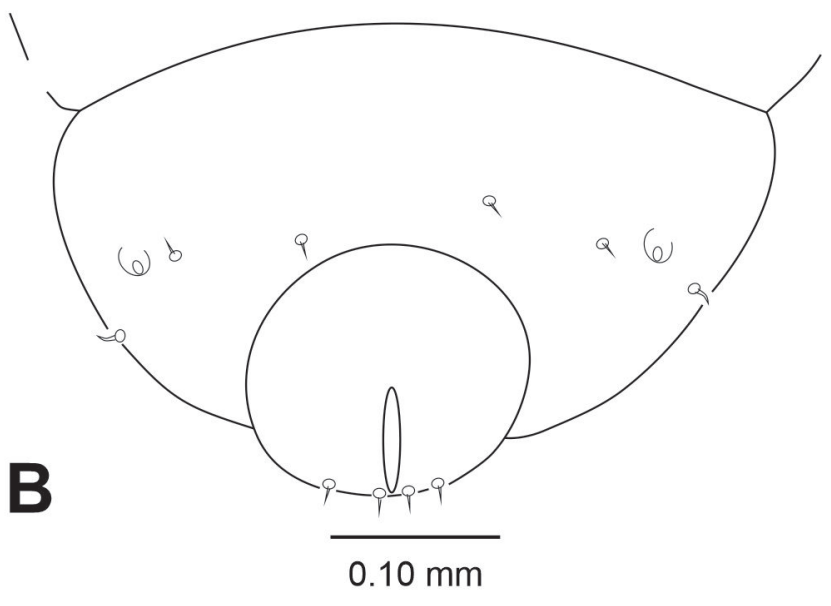

Figure 23. Asphondylia zeyheriae sp. nov., larva, (A) Prothoracic spatula and associated papillae, ventral view, (B) Terminal segment, dorsal view. 

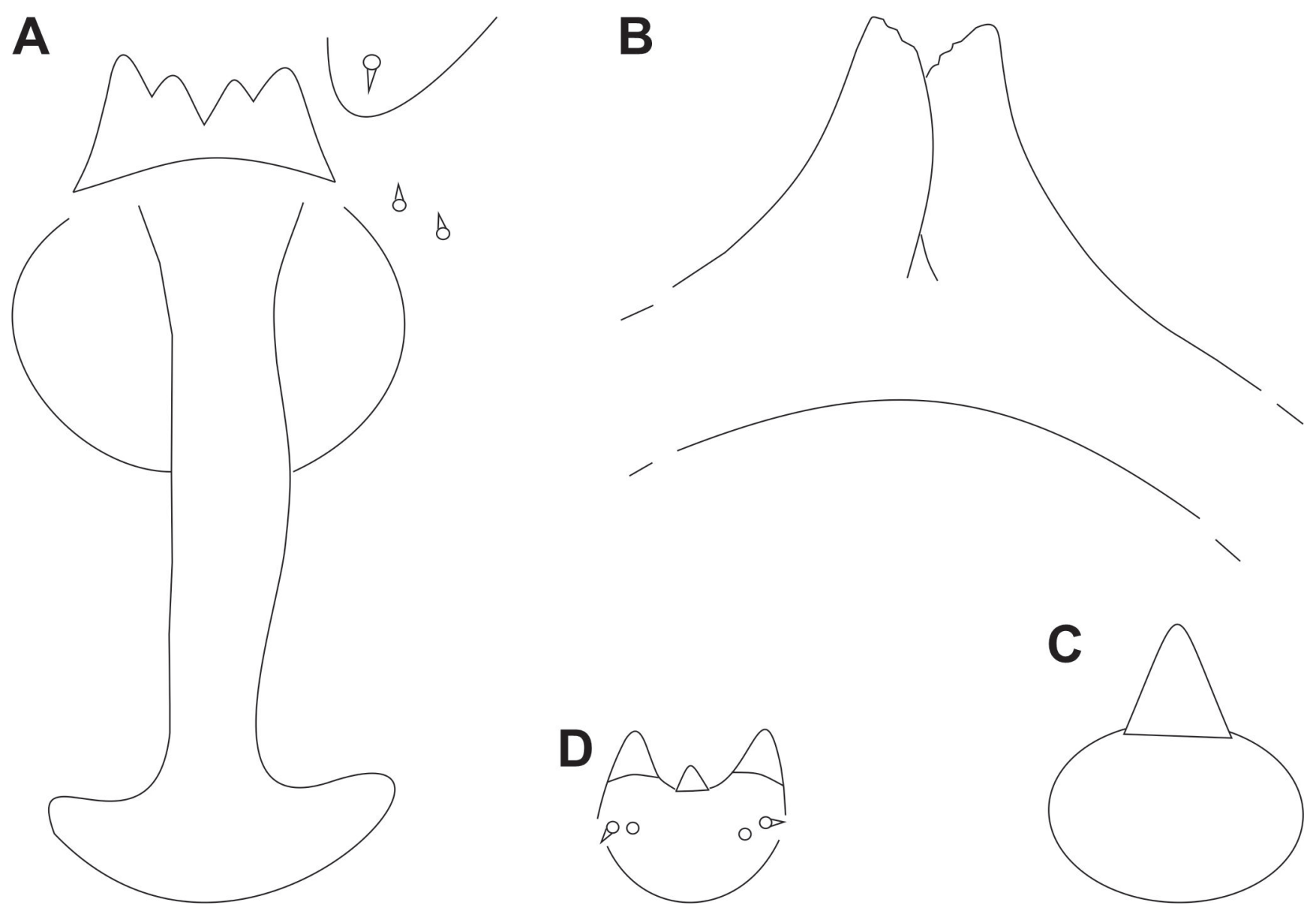

Figure 24. Asphondylia godmanniae Möhn, 1959, (A-B) Larva. (A) Prothoracic spatula and associated papillae, ventral view, (B-D) Pupa, (B) Antennal horn, (C) Upper facial horn, (D) Lower facial horn. All drawings were redrawn from Möhn, 1959.

vae differ mainly in the body length $(1.9 \mathrm{~mm}$ in the former and 3.7-3.9 $\mathrm{mm}$ in the latter), and shape and relative length of the spatula teeth (compare Fig. 23A with Fig. 24A). Pupae of both species differ in body length (2.3-2.4 mm in A. godmaniae and 5.0-6.4 mm in A. zeyheriae) and face integument (sclerotized area between upper and lower facial horns present only in the new species). In both species, the distal part of the antennal horn is longer than the basal part, but in the new species this difference is more accentuated; the upper facial horn is longer and more pointed in A. zeyheriae than in A. godmaniae, and the lower facial horn has the lateral teeth slightly longer than the middle tooth in the new species, while in $A$. godmaniae this difference is greater (compare Figs. 21B-D with Figs. 24B-D).

\section{ACKNOWLEDGMENTS}

To Conselho Nacional de Desenvolvimento Científico e Tecnológico (CNPq) for financial support (\#301481/2017-2).

\section{AUTHORS' CONTRIBUTION}

V.C.M.: Text, slides mounting, descriptions and illustrations. L.A.O.: Collecting of galls on Bauhinia rufa (Fabaceae) in Dores do Indaiá (Minas Gerais), rearing of their gall-inducer in laboratory and gall photography.

\section{REFERENCES}

Cumming, J.M. \& Wood, D.M. 2009. Adult morphology and terminology. In: Brown, B.V.; Borkent, A.; Cumming, J.M.; Wood, D.M.; Woodley, N.E. \& Zumbado, M.A. (Eds.). Manual of Central American Diptera. Ottawa, NRC Research Press. p. 9-50.

Dorchin, N.; Harris, K.M. \& Stireman III, J.0. 2019. Phylogeny of the gall midges (Diptera, Cecidomyiidae, Cecidomyiinae): systematics, evolution of feeding modes and diversification rates. Molecular Phylogenetics and Evolution, 140: 1-15.

Fischer, E.; Theisen, I. \& Lohmann, L.G. 2004. Bignoniaceae. In: Kubitkzki, K. (Ed.). The families and genera of vascular plants. London, Springer. v. 7, p. 9-38.

Funk, V.A.; Sancho, G.; Roque, N.; Kelloff, C.L.; Ventosa-Rodriguez, I.; Diazgranados, M.; Bonifacino, J.M. \& Chan, R. 2014. A phylogeny of the Gochnatieae: Understanding a critically placed tribe in the Compositae. Taxon, 63(4): 859-882.

Gagné, R.J. 1989. The Plant-Feeding Gall Midges of North America. Comstock, Cornell University Press. $356 \mathrm{p}$.

Gagné, R.J. 1994. The gall midges of the Neotropical region. Ithaca, Cornell University Press. $352 \mathrm{p}$.

Gagné, R.J. \& Jaschhof, M. 2021. A Catalog of the Cecidomyiidae (Diptera) of the World, 5. ed. Digital. $813 \mathrm{p}$.

Lohmann, L.G. 2020. Zeyheria. In: Flora do Brasil 2020. Jardim Botânico do Rio de Janeiro. Available: http://floradobrasil.jbrj.gov.br/reflora/ floradobrasil/FB114466. Access: 11/04/2021.

Maia, V.C.; Silveira, F.A.0.; Oliveira, L.A. \& Xavier M.F. 2008. Asphondylia gochnatiae, a new species of gall midge (Diptera, Cecidomyiidae) 
associated with Gochnatia polymorpha (Asteraceae). Zootaxa, 1740: 53-58.

Möhn, E. 1959. Gallmücken (Diptera, Itonididae) aus El Salvador. 1. Teil. Senckenbergiana Biologica, 40: 297-368.

Sancho, G. 2000. Revisión y filogenia de la sección Moquiniastrum Cabrera del género Gochnatia Kunth (Asteraceae, Mutisieae). Fontqueria, 54: 61-122.

Sinou, C.; Forest, F.; Lewis, G.P. \& Bruneau, A. 2016. The genus Bauhinia s.l. (Leguminosae): A phylogeny based on the plastid trnL-trnF region. Botany, 87(10): 947-960.
Urso-Guimarães, M.V. 2018. A new species of Asphondylia (Diptera, (ecidomyiidae) and a key to separate species of the genus associated with Asteraceae from Neotropical region. Papéis Avulsos de Zoologia, 58: 1-7. Urso-Guimarães, M.V. 2019. Description of the male and larva of Schizomyia tuiuiu Urso-Guimarães \& Amorim (Diptera, Cecidomyiidae), new records and a key to Neotropical species of Schizomyia Kieffer. Iheringia, Série Zoologia, 109:1-6. D01

Vaz, A.M.S.F. 2020. Bauhinia. In: Flora do Brasil 2020. Jardim Botânico do Rio de Janeiro. Available: http://floradobrasil.jbri.gov.br/reflora/ floradobrasil/FB22811. Access: 17/03/2021. 